\title{
De novo assembly of the olive fruit fly (Bactrocera oleae) genome with linked- reads and long-read technologies minimizes gaps and provides exceptional $Y$ chromosome assembly
}

\author{
Anthony Bayega ${ }^{1 \dagger}$, Haig Djambazian ${ }^{1 \dagger}$, Konstantina T. Tsoumani ${ }^{2}$, Maria-Eleni Gregoriou ${ }^{2}$, Efthimia Sagri ${ }^{2}$,
} Eleni Drosopoulou ${ }^{3}$, Penelope Mavragani-Tsipidou ${ }^{3}$, Kristina Giorda ${ }^{4}$, George Tsiamis ${ }^{5}$, Kostas Bourtzis ${ }^{6}$, Spyridon Oikonomopoulos ${ }^{1}$, Ken Dewar ${ }^{1}$, Deanna M. Church ${ }^{7}$, Alexie Papanicolaou ${ }^{8}$, Kostas D. Mathiopoulos ${ }^{2^{*}}$ and Jiannis Ragoussis ${ }^{1 *}$ (D)

\begin{abstract}
Background: The olive fruit fly, Bactrocera oleae, is the most important pest in the olive fruit agribusiness industry. This is because female flies lay their eggs in the unripe fruits and upon hatching the larvae feed on the fruits thus destroying them. The lack of a high-quality genome and other genomic and transcriptomic data has hindered progress in understanding the fly's biology and proposing alternative control methods to pesticide use.

Results: Genomic DNA was sequenced from male and female Demokritos strain flies, maintained in the laboratory for over 45 years. We used short-, mate-pair-, and long-read sequencing technologies to generate a combined male-female genome assembly (GenBank accession GCA_001188975.2). Genomic DNA sequencing from male insects using 10x Genomics linked-reads technology followed by mate-pair and long-read scaffolding and gapclosing generated a highly contiguous $489 \mathrm{Mb}$ genome with a scaffold N50 of $4.69 \mathrm{Mb}$ and L50 of 30 scaffolds (GenBank accession GCA_001188975.4). RNA-seq data generated from 12 tissues and/or developmental stages allowed for genome annotation. Short reads from both males and females and the chromosome quotient method enabled identification of Y-chromosome scaffolds which were extensively validated by PCR.

(Continued on next page)
\end{abstract}

\footnotetext{
* Correspondence: kmathiop@bio.uth.gr; ioannis.ragoussis@mcgill.ca

${ }^{\dagger}$ Anthony Bayega and Haig Djambazian are co-first authors

${ }^{2}$ Department of Biochemistry and Biotechnology, University of Thessaly, Biopolis, 41500 Larissa, Greece

'McGill University and Genome Quebec Innovation Centre, Department of Human Genetics, McGill University, Montreal, Canada

Full list of author information is available at the end of the article
}

(c) The Author(s). 2020 Open Access This article is licensed under a Creative Commons Attribution 4.0 International License, which permits use, sharing, adaptation, distribution and reproduction in any medium or format, as long as you give appropriate credit to the original author(s) and the source, provide a link to the Creative Commons licence, and indicate if changes were made. The images or other third party material in this article are included in the article's Creative Commons licence, unless indicated otherwise in a credit line to the material. If material is not included in the article's Creative Commons licence and your intended use is not permitted by statutory regulation or exceeds the permitted use, you will need to obtain permission directly from the copyright holder. To view a copy of this licence, visit http://creativecommons.org/licenses/by/4.0/ The Creative Commons Public Domain Dedication waiver (http://creativecommons.org/publicdomain/zero/1.0/) applies to the data made available in this article, unless otherwise stated in a credit line to the data. 
(Continued from previous page)

Conclusions: The high-quality genome generated represents a critical tool in olive fruit fly research. We provide an extensive RNA-seq data set, and genome annotation, critical towards gaining an insight into the biology of the olive fruit fly. In addition, elucidation of Y-chromosome sequences will advance our understanding of the $Y$ chromosome's organization, function and evolution and is poised to provide avenues for sterile insect technique approaches.

Keywords: Olive fruit fly genome, Bactrocera oleae, Linked reads, Long reads, Y chromosome assembly, Insect developmental genes

\section{Background}

Some animals have always been "more equal" than others. ${ }^{1}$ For many researchers, working on anything ranging from classical genetics to developmental biology to modern genomics, the "most equal" animal has been Drosophila melanogaster. Despite Drosophila's insignificant agricultural or medical importance, it became, in 2000, the first complex eukaryote whose genome was sequenced and assembled [1]. More important insect genomes, like that of the malaria mosquito Anopheles gambiae, followed soon after [2]. However, non-model insects or insects with less important public health or global agricultural impact had a much harder time having their whole genomes sequenced. This held back several advances that would be based on understanding their genomes, including tools for developing alternative pest control methods. Gradually, advances in DNA sequencing technologies that dramatically reduced the cost and time to sequence an organism's entire genome made sequencing of numerous insect genomes a reality. In 2011, the "i5k" initiative was launched to provide the genomic sequences of 5000 insect or related arthropod species [3]. In this project, the onus was placed on individual labs with a specific interest in these genomes to organize the sequencing, analysis, and curation of their genomes [4]. Eight years later the target is still far from being achieved. As of March 2019, only 1219 insect genomes had been registered in the National Center for Biotechnology Information (NCBI) and only 401 of them have had at least a draft genome assembly [5].

The goal of sequencing 5000 insect genomes was not put as a mere technological challenge. Sequencing information can enormously help the understanding of insect biology as well as provide insights for environmentally friendlier means of control. For example, accurate genome sequence information is now the basis for precise CRISPR-based genetic manipulation and genome editing (e.g., Kyrou et al. [6]), or for designing RNAibased species-specific and eco-friendly insecticides (for a recent review see Vogel et al. [7]). Furthermore, the genomic diversity of ecotypes, geographical isolates and

\footnotetext{
${ }^{1}$ George Orwell, Animal Farm
}

related species can be combined with genome-wide association studies (GWAS) and reveal the genetic components of certain traits and adaptations such as insecticide resistance $[8,9])$, geographical polymorphism $[10,11])$ or host adaptation [12]. Despite this importance, insect whole genome sequencing (WGS) projects are not advancing at the anticipated pace. Firstly, small physical insect sizes might not allow enough quantities of DNA to be isolated from a single individual. Secondly, high population polymorphism and/or difficulty to breed for genome homozygosity renders genome assembly efforts particularly difficult [13]. Therefore, it is critical to establish methodological approaches that will allow the de novo sequencing of insect genomes at high quality and low cost if the i5k target is to be achieved.

The ideal sequencing approach should provide very long reads (in order of megabases, $\mathrm{Mb}$ ) with single basepair resolution, very low error rate, and low cost. However, no such platform currently exists. Short-read sequencing technologies, such as 'single nucleotide fluorescent base extension with reversible terminators [14]' commonly referred to as Illumina sequencing (Illumina Inc.), deliver massive numbers of relatively cheap short (50-300 bp) high quality reads but de novo genome assemblies from such technologies are often fragmented. On the other hand, long-read sequencing technologies such as nanopore sequencing from Oxford Nanopore Technologies (ONT) and Single Molecule Real-Time (SMRT) sequencing from Pacific Biosciences Inc. (PacBio) which deliver long reads have relatively low throughput and high raw-read error rates. However, assemblies from these technologies are much more contiguous yielding completely closed genome assemblies for small organisms like prokaryotes [15]. To benefit from the pros of each sequencing technology, hybrid approaches that aim to sequence organisms using different approaches and then combine the data, either at the level of error correction of reads or scaffolding and gapclosing of assemblies, are increasingly widely applied (reviewed elsewhere [16]). Hybrid genome assemblies have shown more accuracy and contiguity [15, 17], and are now a preferred approach to de novo genome assembly. 
The linked-reads technology $[18,19]$ from 10x Genomics (CA, USA) is a relatively new genomic library preparation approach. Conceptually, a single ultra-long DNA fragment is captured into an oil emulsion droplet (also called GEM or partition) and sampled along the length of the fragment using oligonucleotides bearing the same molecular barcode for each partition. Pooling and Illumina sequencing of all barcoded oligos and computationally linking all oligos taken from the same DNA molecule using the bespoke Supernova assembly tool [20] provides a new powerful approach for using short-read technologies in de novo genome assembly. This method has previously been applied to insect genomes with varying levels of success [21, 22]. This is probably partly because this entire methodology is optimized around human genomes and genomes of similar size, while for genomes of significantly smaller sizes, optimization of assembly parameters is needed [20].

In the current manuscript we present several technological advances that were developed in order to sequence the entire genome of a non-model organism but one of high agricultural significance, the olive fruit fly (Bactrocera oleae), whose genome size was initially estimated to be $322 \mathrm{Mb}$ using qPCR [23]. The olive fruit fly belongs to the Tephritidae family of insects, a family that contains some of the most important agricultural pests world-wide, such as the Mediterranean fruit fly (medfly, Ceratitis capitata), the oriental fruit fly (Bactrocera dorsalis), the Mexican fruit fly (Anastrepha ludens), the Australian Queensland fruit fly (Bactrocera tryoni) and others. Olive fruit flies are the major pest of wild and commercially cultivated olives trees causing an estimated annual damage of USD 800 million [24, 25], since chemical insecticides do not fully protect a tree from being infested. Despite its economic importance in olive producing countries, several peculiarities of the olive fruit fly's biology (e.g., difficulty in rearing, high natural homozygosity, lack of phenotypic mutations) made the development of classical genetics tools an impossible task. More recently, however, the olive fruit fly has been the subject of several molecular and transcriptomics studies [26-28] (Reviewed in Sagri et al. [29]).

Another particularity of the olive fruit fly is the fact that it possesses a very small Y chromosome [30, 31], karyotypically appearing as the $\sim 4 \mathrm{Mb}$ dot chromosome IV of $D$. melanogaster [32]. Among organisms that employ an X-Y chromosome system, as does the olive fruit fly, the $\mathrm{Y}$ chromosome has been notoriously difficult to assemble due to its heterochromatic and repetitive nature. For example, $80 \%$ of the Drosophila melanogaster Y chromosome is made up of repeats [33]. In most genome sequencing projects, the $\mathrm{Y}$ chromosome sequence is fragmented into many small, unmapped scaffolds [34]. Additionally, only a few genes reside on the $\mathrm{Y}$ chromosome and most of them are characterized by the presence of small exons, gigantic introns, and very little conservation among species even of the same family [35]. Therefore, Y chromosome assembly presents a unique challenge. In the olive fruit fly, the Y chromosome encompasses the male determining factor, $M$, that had remained elusive for over 30 years [36]. The $M$ factor is the initial switch of the sexdetermining cascade in tephritids, a switch that has been speculated to differ from the one used by the model dipteran Drosophila (for a review see [37]). The $M$ factor has recently been identified in the medfly and a few other tephritids, including the olive fruit fly [38], but the details of the sex determination cascade remain unclear. Unraveling this cascade and identifying other genes that reside on the Y chromosome, probably involved in male fertility, will shed light on the evolution of a major developmental pathway in most animals, as well as the evolution of the sex chromosomes themselves $[39,40]$.

Here, we describe the whole genome sequence of the olive fruit fly, generated as a hybrid assembly using the 10x Genomics linked-reads assembly as the backbone followed by scaffolding and gap-filling with Illumina mate-pair reads, and long-reads from PacBio and ONT. This genome has a scaffold N50 of $4.69 \mathrm{Mb}$ and L50 of 30 making it one of the most contiguous Tephritidae genomes in the current NCBI genome catalogue. We also identified $\mathrm{Y}$ chromosome-specific scaffolds and present the first assembly of the B. oleae Y chromosome that will be instrumental in the elucidation of the regulation of the $M$ factor and the structure and evolution of the entire $\mathrm{Y}$ chromosome. We also provide 12 short-read RNA-seq datasets from different tissues and/or development stages which add extensive characterization of this organism.

\section{Results}

In order to generate a high-quality genome assembly of the olive fruit fly we undertook a multistep process that consisted of different sequencing and assembly approaches (Fig. 1, Supplementary Figure S1). First, we generated sequence data using short-read and long-read sequencing platforms that was used to generate a hybrid assembly (GenBank accession GCA_001188975.2). We then used the 10x Genomics linked-reads technology to generate an independent haplotype-resolved assembly. The final steps involved scaffolding and gap-closing of the $10 \mathrm{x}$ assembly using mate-pair and long-reads and then finally polishing to generate the final assembly (GenBank accession GCA_001188975.4). The steps undertaken and the resulting assemblies are detailed below.

\section{Genome assembly using Illumina paired-end, mate-pair and PacBio reads}

Our initial assembly was performed using two Illumina short insert paired-end (PE) libraries made separately 


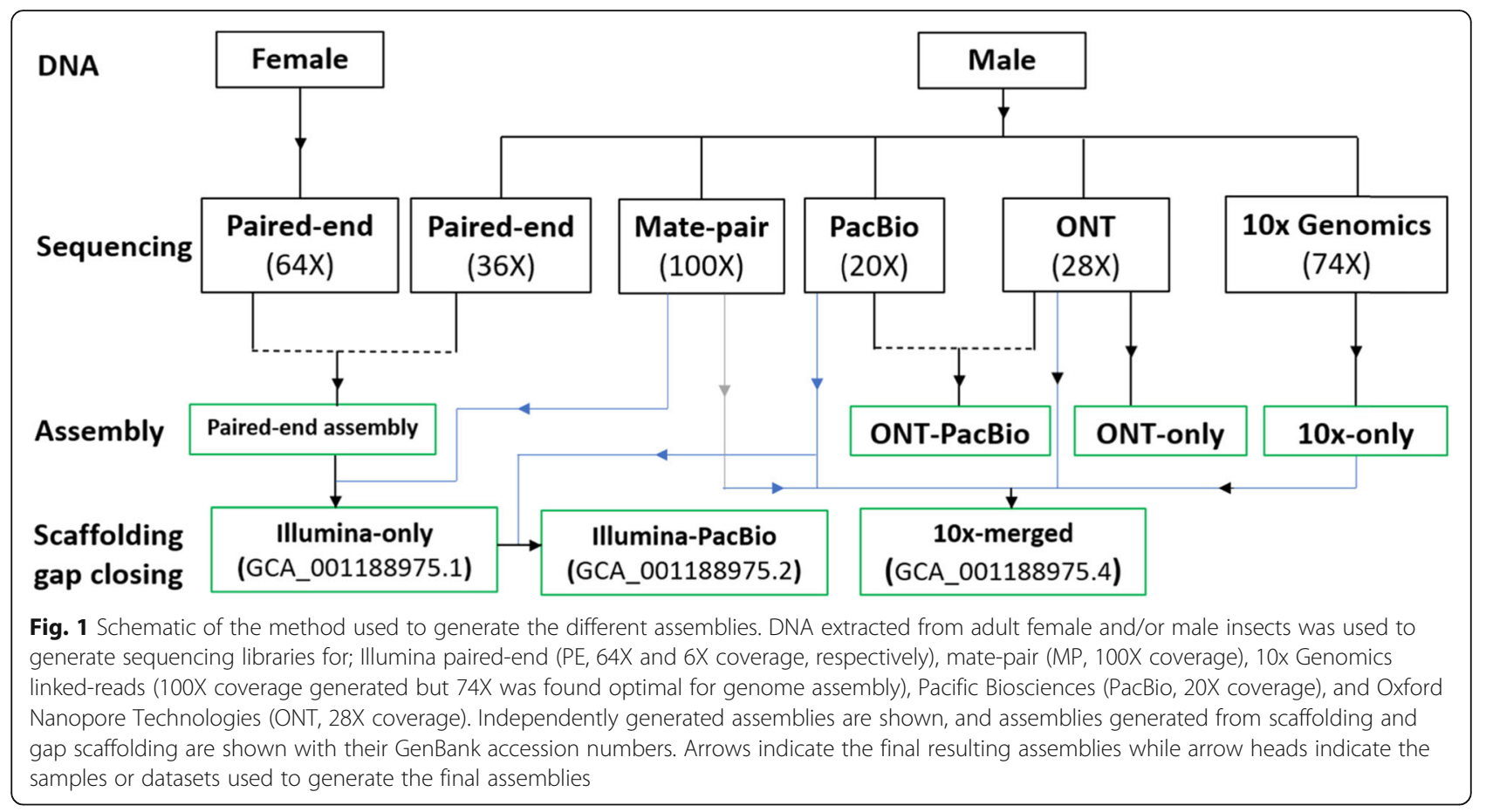

from male and female flies, the sequencing of which yielded $36 \mathrm{X}$ and $61 \mathrm{X}$ theoretical coverage, respectively (see Supplementary Table S1). Male and female reads were assembled together using a short-read assembler, Ray [41], with a kmer of $k 41$ which produced the largest scaffold. The assembly was further scaffolded with $100 \mathrm{X}$ coverage from three mate-pair (MP) libraries using SSPACE [42], and then gap-filled using 20X coverage of reads generated with SMRT technology from Pacific Biosciences (PacBio). This resulted in a final assembly that was submitted to NCBI (GenBank assembly accession: GCA_001188975.2). The submitted assembly had a total length of $471,780,370$ bases with a scaffold N50 length of $139,566 \mathrm{bp}$ reached with 474 scaffolds (Table 1, Supplementary Table S2). GCA_001188975.2 was also submitted to i5k [3].

\section{Utilization of linked-reads to generate a Bactrocera oleae assembly}

The 10x Genomics platform which generates linkedreads has great potential to yield high quality assemblies in terms of base accuracy, contiguity, and phasing. High molecular weight DNA was extracted from male 'Demokritos' strain of the olive fruit fly which has been a lab strain for over 45 years. This strain has been maintained in our lab for over 15 years with no addition of wild flies. Unlike the C. capitata genome [44] that required inbreeding of the ISPRA strain for 20 generations which resulted in low heterozygosity $(0.391 \%)$, the Demokritos olive fruit fly strain used in the current research was already of low heterozygosity $(0.401 \%$, Supplementary Figure S2). This is due to the huge bottleneck that the olive fruit fly undergoes during domestication [45], the large number of years that the Demokritos strain has spent in laboratory conditions (> 45) and, probably, other reasons that have to do with the biology of the insect (e.g., strict monophagy of the larva). Linked-reads library preparation (done at 10x Genomics, San Francisco, CA, USA) and sequencing resulted in 100X coverage worth of data which was assembled using the bespoke Supernova assembler. Because genome assembly with 10x Genomics data was only optimized for human genomes [20], we derived our optimized parameters. Specifically, we performed several rounds of genome assemblies varying the coverage depth and number of partitions and compared the resulting NG50. The assembly NG50 increased with increasing coverage up to a peak above which the assembly NG50 dropped

Table 1 Statistics for the main B. oleae genome assemblies generated

\begin{tabular}{llllllll}
\hline GenBank Accession & Name & \# scaffolds/contigs & Total length (Mb) & Largest contig (Mb) & N50 (Mb) & L50 & \# N's per 100 kb \\
\hline GCA_001188975.2 & Illumina-PacBio & 36,198 & 472 & 5.1 & 0.14 & 474 & $10,853.91$ \\
GCA_001188975.4 & 10x-All & 39,141 & 489 & 19.4 & 4.69 & 30 & 5493.82 \\
\hline
\end{tabular}

Quality metrics were generated using Quast [43]. N50 value is the scaffold/contig length at which half of the genome is contained in scaffolds/contigs at or above that length. L50 is the number of contigs needed to reach N50 
for all partitions tested (Fig. 2a). Increasing coverage had the opposite effect on genome LG50 (Fig. 2b). The best assembly was obtained with $74 \mathrm{X}$ coverage and 500,000 partitions which corresponded to 331 reads per partition. The optimized parameters (number of partitions to use, reads per partition, and coverage) were used to generate an assembly of $434.81 \mathrm{Mb}$ with a scaffold N50 of $2.16 \mathrm{Mb}$, with the largest scaffold stretching $12 \mathrm{Mb}$. The L50 was only 44 (Supplementary Table S3, Supplementary Figure S3). This assembly is here referred to as $10 x$-only. Using this assembly as the backbone, several scaffoldings were performed to increase genome contiguity.

Scaffolding and gap-closing of the linked-reads assembly We explored the effectiveness of combining the 10xonly assembly with short-reads and long-reads. Oxford Nanopore technologies (ONT) and Pacific Biosciences currently generate the longest raw reads of any commercially available DNA sequencers with ONT having no theoretical limits [46]. This provides potential to significantly increase assembly contiguity. High molecular weight DNA was extracted from a pool of adult male flies and used to prepare ONT and PacBio sequencing libraries, the sequencing of which resulted in a theoretical coverage of $28 \mathrm{X}$ and $20 \mathrm{X}$, respectively. The ONT reads had an N50 of $11 \mathrm{~kb}$ with the longest read generated being $780 \mathrm{~kb}$. The short and long reads enabled scaffolding and gap-closing of the 10xonly assembly (see Supplementary Table S3 and Supplementary Figure S3 for a summary of the results). Using SSPACE, mate-pair sequences were used to scaffold the 10x-only assembly. This had a noticeable improvement on the $10 x$-only assembly increasing the $\mathrm{N} 50$ from 2.16 to $3.26 \mathrm{Mb} \quad(51 \%$

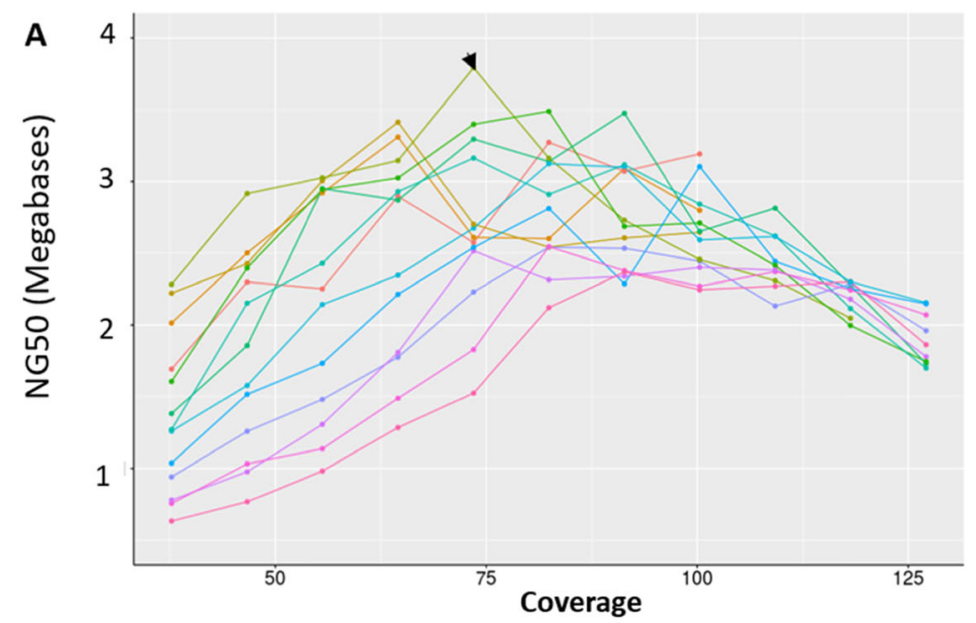

partitions

$-200000$

$\rightarrow 300000$

$\rightarrow 400000$

$\rightarrow 500000$

$\rightarrow 600000$

$\rightarrow 700000$

$\rightarrow 800000$

$\rightarrow 900000$

$\rightarrow 1000000$

B

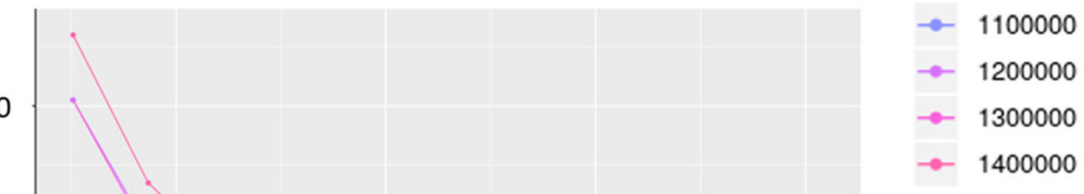

윰

80

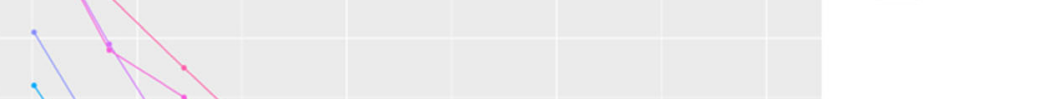

60

40

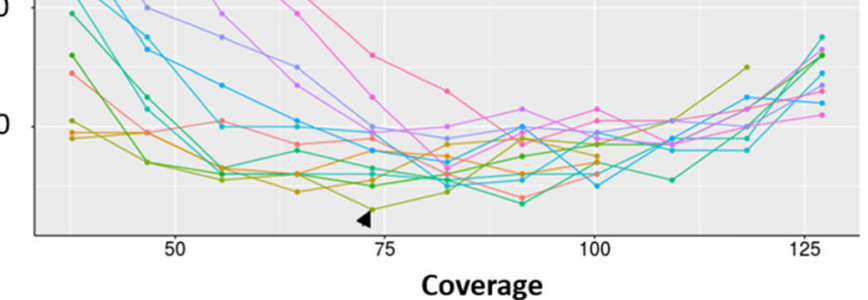

Fig. 2 Optimization of number of partitions and coverage for the Supernova assembler. Different number of partitions were randomly selected using the partition (GEM) barcodes while also varying the number of reads per partition to optimize the coverage. These were provided as input for the assembler. For each resulting assembly the NG50 length and LG50 count were calculated with genome size assumed to be 320 Mb [23]. NG50 value is the scaffold/contig length at which half of the genome $(\sim 160 \mathrm{Mb})$ is contained in scaffolds/contigs at or above that length. LG50 is the number of contigs needed to reach N50. Arrow heads indicate optimized parameters 
increase) at the expense of including gaps between scaffolded contigs (gaps increased from 3543.94 to 10, $744.3 \mathrm{~N}$ 's per $100 \mathrm{~kb}$ ). Scaffolding the $10 \mathrm{x}$-only assembly with PacBio reads (20X coverage) using PBJelly increased the 10x-only scaffold N50 from 2.16 to $3.77 \mathrm{Mb}$ ( $74 \%$ increase) and reduced the L50 to 32 scaffolds. Scaffolding the $10 x$-only assembly using ONT reads (28X coverage) had the biggest improvement on contiguity. The scaffold N50 more than doubled from 2.16 to $4.59 \mathrm{Mb}$ ( $112 \%$ increase) and the L50 was reduced from 44 to 29 scaffolds. Further, the ONT reads increased the largest scaffold from $12 \mathrm{Mb}$ to $19.3 \mathrm{Mb}$. Scaffolding with either PacBio or ONT had similar effects on assembly gaps (reducing from 3544 to 3538 and $3532 \mathrm{~N}$ 's per $100 \mathrm{~kb}$, respectively).

The final assembly was generated by combining all technologies. Scaffolding the 10x-only assembly first with mate-pairs then PacBio followed by ONT produced the highest contiguity. The final assembly was polished using Pilon and submitted to NCBI with assembly name "MU_ Boleae_v2" (GenBank accession; GCA_001188975.4). This is the most contiguous $B$. oleae genome assembly to date (see Supplementary Figure S4 for comparison to the previous assembly). The total assembly size is $488.86 \mathrm{Mb}$, with scaffold N50 of $4.69 \mathrm{Mb}, 36,198$ total scaffolds, and scaffold L50 of 30 (Table 1). This genome size is slightly larger than the $446 \mathrm{Mb}$ predicted using kmer analysis [47] and significantly larger than $322 \mathrm{Mb}$ predicted by qPCR [23]. This genome size is similar to other closely related species (Ceratitis capitata, $479 \mathrm{Mb}$ [44]; Bactrocera dorsalis, 414 $\mathrm{Mb}$; Zeugodacus cucurbitae, 374 Mb). Generally, insect genome sizes differ greatly from $68.5 \mathrm{Mb}$ (Midge, Clunio tsushimensis) to $16.5 \mathrm{~Gb}$ (Mountain grasshopper, Podisma pedestris), with median of $498.8 \mathrm{Mb}$ [48]. Dipteran insects, however, have smaller genomes ranging from $68.5 \mathrm{Mb}$ (Midge, Clunio tsushimensis) to $1.8 \mathrm{~Gb}$ (Mosquito, Aedes zoosophus), median $224.9 \mathrm{Mb}$ [48]. The olive fruit fly genome at $485 \mathrm{Mb}$ is about the median insect size and about twice the median Dipteran genome size.

\section{Identification of sex chromosome sequences and $\mathrm{Y}$ chromosome assembly}

In order to find putative $\mathrm{X}$ or $\mathrm{Y}$ chromosome scaffolds we used the Chromosome Quotient (CQ) method [49]. The CQ reflects the median ratio of female to male reads coverage when these reads are separately aligned to a male genome assembly. The CQ values will cluster around zero, one, or two for $\mathrm{Y}$, autosome, and X scaffolds, respectively. Using the repeat masked version of the final assembly (GCA_001188975.4), which was generated from male olive fruit fly DNA, male and female short Illumina reads (40X coverage of each) were independently mapped. Considering only the scaffolds with a CQ of 0 , we obtained a total length of putative $\mathrm{Y}$ - chromosome of $3.9 \mathrm{Mb}$ with 873 scaffolds (Fig. 3a). We similarly determined putative Y-chromosome scaffolds from other assemblies and compared them (Supplementary Figure S5, Supplementary Table S4). The GCA 001188975.4 Y scaffolds showed high contiguity with a scaffold N50 of $60 \mathrm{~kb}$ and the largest scaffold being 318 $\mathrm{kb}$. The size of our assembled B. oleae $\mathrm{Y}$ chromosome at $3.9 \mathrm{Mb}$ is very similar to the predicted size of $4 \mathrm{Mb}$ [32] and thus likely captures most of it. The $\mathrm{X}$ chromosome scaffolds identified in the GCA_001188975.4 assembly totaled $6 \mathrm{Mb}$.

\section{Validation of Y-chromosome specific scaffolds}

To validate the $\mathrm{Y}$ scaffolds identified using the CQ method, 85 primer pairs (see Supplementary Table S5), chosen from the largest scaffolds, were designed to amplify regions of the different Y-linked scaffolds by polymerase chain reaction (PCR) using either male or female genomic DNA as template. When a primer pair resulted in the amplification of the expected size band with male genomic DNA only, we concluded that its corresponding scaffold was Y-specific. However, it was expected that some primers might represent homologous regions between $\mathrm{X}$ and $\mathrm{Y}$ chromosomes and thus have a product both in male and female samples, albeit at a lower level in the females. Partial homology with autosomal sequences was also expected. Quantitative real time PCR (qPCR) offers a much more precise method to detect such differences. Therefore, lower male qPCR cycle-threshold (Ct) amplification values than female should indicate that the respective primer pair corresponded to a Y-specific scaffold. Nine primer pairs gave no amplification, 11 gave ambiguous results and require further examination, while 30 equally amplified male and female gDNA. A total of $1.7 \mathrm{Mb}$ out of $3.9 \mathrm{Mb}$ in GCA_001188975.4 assembly was thus confirmed as Y-chromosome (Supplementary Figure S6). To further validate scaffolds potentially containing $\mathrm{Y}$ chromosome sequence, we used the $\mathrm{Y}$ chromosome Genome Scan method (YGS, [51]) which retrieved 1196 scaffolds totaling $3.9 \mathrm{Mb}$. Of these scaffolds, 271 scaffolds totaling $2.7 \mathrm{Mb}$ or $68 \%$ of putative Y chromosome had also been identified using the CQ method. Further, the scaffolds identified using YGS method contained all the PCR confirmed scaffolds. The Y chromosome, however, remains difficult to assemble with orthogonal methods yielding slightly differing results.

\section{Generation of chromosome markers and scaffolds assignment to chromosomes}

The olive fruit fly has well-characterized cytogenetic maps derived from polytene chromosomes [52], which enables the determination of the exact position of scaffolds containing specific markers. Further, scaffolds 
A

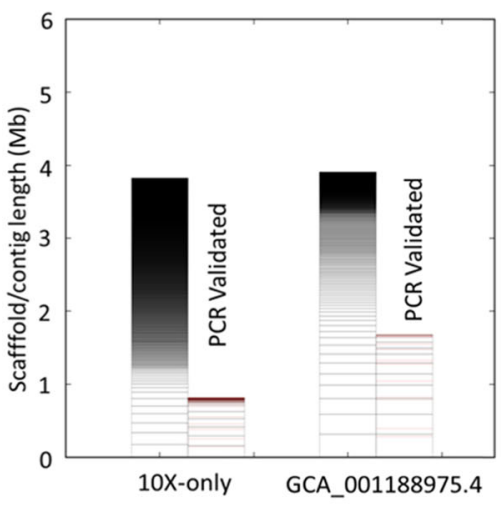

B

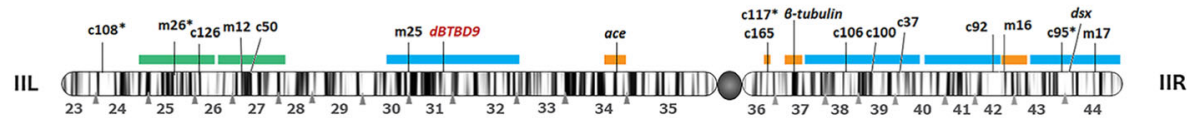

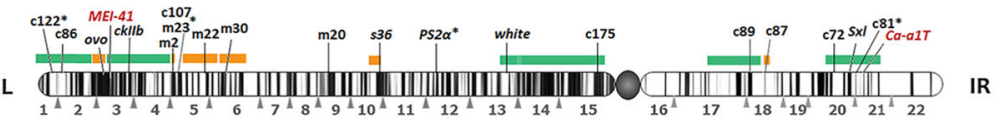
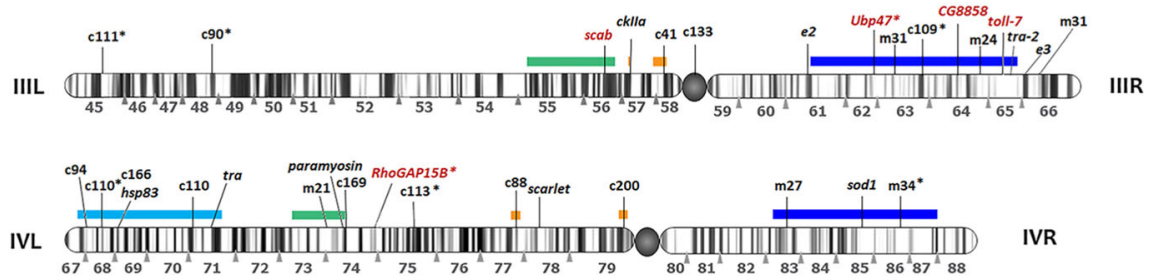

VL
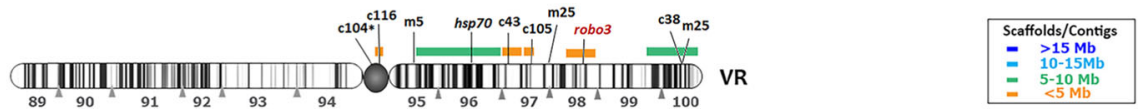

Fig. 3 B. oleae polytene chromosomes mapping of molecular markers $Y$ chromosome assembly. a Plot showing $Y$ chromosome scaffolds/contigs identified in 2 different assemblies (Supplementary Table S4). The Chromosome Quotient (CQ) method [49] was used to identify Y chromosome scaffolds. The scaffolds/contigs are ordered from longest at the bottom to shortest at the top. For each assembly the total scaffolds/contigs are shown in left bars while the PCR validated scaffolds/contigs are the right bars. The approximate location of the PCR primer on the scaffold/contig is shown in pink. $\mathbf{b}$ Schematic representation of $B$. oleae polytene chromosomes including all mapped markers (tags) and the scaffolds assigned to chromosomes. Previously and currently mapped markers are indicated with black and red letters, respectively, above chromosomes. Colored horizontal bars above chromosomes indicate scaffolds/ contigs in the GCA_001188975.4 assembly that were localized to chromosomes using mapped markers. More than one tags on a specific scaffold is informative of its physical orientation. m\#\# corresponds to microsatellite markers number \#\#; c\#\# corresponds to EST marker number \#\# [26, 50]; newly mapped genes in the current study are presented in full names or abbreviations (Supplementary Table S6); "*" indicates the tags that were not found on the anchored contig or gave ambiguous alignment results. The centromere is shown as a filled circle. (see Supplementary Table S7 for detailed information)

containing more than one mapped marker can be oriented on the chromosomes. We, therefore, used already mapped and newly generated molecular markers in order to position sequenced scaffolds on the chromosomes. The markers used included 35 expressed sequence tags (ESTs) [26], 16 microsatellites [53], and 19 previously localized heterologous genes [50, 52, 54] providing 70 tags in total. As part of the current work we generated 9 new markers (Supplementary Table S6) and mapped their position on salivary gland polytene chromosomes by in situ hybridization (Fig. 3b). All 79 tags were aligned using Minimap2 [55] in splice-aware mode and also BLAST'ed. against the GCA_001188975.4 genome in order to assign scaffolds/contigs to chromosomal arms. However, 25 tags gave ambiguous alignment results and were not used further. The remaining 54 tags allowed the physical mapping of 36 contigs with a total length of $200 \mathrm{Mb}$, corresponding to $41 \%$ of the total genome size (Fig. 3b, Supplementary Table S7). Among the 36,10 scaffolds totaling $106 \mathrm{Mb}$ contained more than 1 marker and could thus be oriented. Addition of the $\mathrm{X}$ and $\mathrm{Y}$ chromosome scaffolds totaling 6 and $4 \mathrm{Mb}$, 
respectively, that were identified using the CQ method brought the total percentage of the genome assigned to chromosomes to 43\% (Fig. 3b, Supplementary Figure S7).

\section{Evaluation of assembly completeness}

We evaluated genome completeness using 3 metrices; genome size, alignment of RNA-seq data, and recovery of basic universal single copy orthologs (BUSCOs [56]). The frequence of k-mers of length $=23$ bases was calculated using BBMAP [57] followed by genome size estimation using GenomeScope [58] (Supplementary Figure S2). The B. oleae genome size was estimated at $439.8 \mathrm{Mb}$. This was close to the final genome assembly size of $489 \mathrm{Mb}$. We generated RNA-seq data from 12 different tissues/stages and aligned them to the GCA_001188975.4 assembly. RNA-seq data alignment rates ranged from 85 to $96 \%$ (Supplementary Figure S8), which is similar to the expected ranges of 70 and $90 \%$ [59]. Perhaps owing to the low heterozygosity, the diploid genome had similar alignment rates to the GCA_001188975.4 assembly which is a single haplotype (Supplementary Figure S8). Nonetheless, we separately provide the second haplotype (SRA index SRR9678778). BUSCOs analysis showed that across the 4 lineages analysed; Eukaryota, Arthropoda, Insecta, and Diptera, 99.3, 99.2, 99.2, and 98.1\% of the genes surveyed were captured in the GCA_001188975.4 assembly (Supplementary Figure S9). The complete Diptera BUSCOs recovered in the GCA_001188975.4 assembly (98.1\%) were higher than the previous assembly GCA_001188975.2 (95.6\%) showing an improvement in assembly quality.

\section{Identification of symbiont derived sequences}

Sequences that belong to bacterial contaminants or symbionts in the GCA_001188975.4 assembly were identified using a similar approach applied to the Mediterranean fruit fly [44]. We identified small fragments that displayed homology with Wolbachia sequences. The biggest fragment identified was $831 \mathrm{bp}$ in length exhibiting a similarity of $89.2 \%$ with an ankyrin from the wMau Wolbachia strain. In total 14 fragments were identified with a size range from 259 to $855 \mathrm{bp}$. No Cardinium and Spiroplasma sequences were present either in the raw dataset or in the assembled contigs.

Our second approach using bacterial complete and draft genomes deposited in NCBI (assessed June 2019) revealed the presence of sequences affiliated mainly with Agrobacterium rhizogenes, Deftlia sp., and Agrobacterium tumefaciens which were found to be present in 17.5, 15.9 and $8 \%$ of all scaffolds, respectively. Most of the sequences identified $(84.6 \%)$ had a length of 100 to $2500 \mathrm{bp}$. Eight alignments were spanning more than $20,000 \mathrm{bp}$. Alignments smaller than 100 bases were considered as noise and were not included in the analysis. The percentage of sequence similarity was between 100 and $65 \%$ with $43.7 \%$ exhibiting a similarity between 90 and $100 \%$. It's worth noting that no sequences of the olive fruit fly symbiont Candidatus Erwinia dacicola were identified which confirms previous reports that this symbiont was lost upon the laboratory domestication and the artificial rearing of this insect pest species [60]. Nevertheless, trimming or removal of scaffolds with evidence of bacterial DNA was guided by NCBI assembly quality check. NCBI quality control and contamination check identified 134 scaffolds/contigs totaling $147 \mathrm{~kb}$ with bacterial origin which were removed. A further 981 scaffolds/contigs totaling $3.92 \mathrm{Mb}$ were suppressed due to possession of bacterial gene models.

\section{Transposable element identification and annotation}

Discovered in the late 1940s in maize [61], transposable elements (TEs) have since been found in almost all eukaryotic organisms surveyed except for Plasmodium falciparum [62]. The highest TE subdivision, Class, comprises 2 groups; Class I and Class II. Class I comprises retrotransposons which utilize a 'copy-andpaste' mechanism of transposition with an RNA intermediate while Class II comprises DNA transposons that utilize a 'cut-and-paste' transposition mechanism with a DNA intermediate. The major orders in Class I are; LTR, DIRS, PLE, LINE, and SINE. Major orders in Class II are; TIR, Crypton, Helitron, and Maverick. TEs are further subdivided down to subfamily level. Virtually all these types of TEs are found in insect genomes with Class I elements being more predominant [63]. LTR for example are the most predominant in $D$. melanogaster, followed by LINEs, and TIR [64, 65]. In insects, TEs play a role in mutagenesis, inter and intra-chromosomal rearrangements, evolution of sex chromosomes, and genomic adaptation (reviewed in [60]). Discovery methods of TEs can be divided into 2; those that rely on raw sequence reads and those that rely on an assembled genome [66]. Due to the challenges in detecting and annotating TEs, combining tools has been shown to improve detection $[67,68]$. We used the PiRATE TE detection pipeline [67] (Supplementary Figure S10), which includes 9 genome based TE identification tools, to derive a TE library. The TE library was classified using PASTEC [69] and then used to annotate and mask the genome using TEannot [70] and RepeatMasker, respectively.

PASTEC classification of the repeat library (Table 2) showed that Class II TEs were most numerous of all repeat elements $(45 \%)$. This contrasts with C. capitata where Class I are the most numerous (55.9\% of TEs) [44]. Terminal inverted repeat (TIR) transposons subclass, which includes the Tc1-mariner superfamily, was most numerous accounting for $29 \%$ of all TEs. However, the B. oleae and C. capitata percentages of LTR elements ( $15.9 \%$ vs $15.7 \%$, of all TEs respectively) 
Table 2 Classification of transposable elements (TE) identified in B. oleae genome

\begin{tabular}{|c|c|c|c|}
\hline Class & Order & Number & Percentage \\
\hline \multirow[t]{10}{*}{ Class I } & DIRS & 30 & 0.06 \\
\hline & LARD & 1111 & 2.12 \\
\hline & LINE & 7802 & 14.85 \\
\hline & LTR & 8339 & 15.88 \\
\hline & PLE & 5 & 0.01 \\
\hline & SINE & 206 & 0.39 \\
\hline & TRIM & 831 & 1.58 \\
\hline & No order & 78 & 0.15 \\
\hline & Several orders & 11 & 0.02 \\
\hline & Total & 18,413 & 34.99 \\
\hline \multirow[t]{7}{*}{ Class II } & Helitron & 7700 & 14.66 \\
\hline & MITE & 113 & 0.21 \\
\hline & Maverick & 133 & 0.25 \\
\hline & TIR & 15,301 & 29.13 \\
\hline & No order & 481 & 0.92 \\
\hline & Several orders & 21 & 0.04 \\
\hline & Total & 23,749 & 45.13 \\
\hline \multicolumn{2}{|c|}{ Simple Sequence Repeats } & 100 & 0.19 \\
\hline \multicolumn{2}{|c|}{ No category } & 10,361 & 19.73 \\
\hline \multicolumn{2}{|l|}{ Total } & 52,623 & 100 \\
\hline
\end{tabular}

Nine de novo and similarity based TE identification tools included in the PiRATE pipeline [67] were used to generate a library of TE followed by classification using PASTEC [69]

and DNA transposons $(45.15 \%$ vs $44.1 \%$, of all TEs respectively) are similar [44]. C. capitata genome was also assembled using long-reads and thus, repeated regions should be fairly well captured.

Genome repeat masking using the derived TE library and RepeatMasker showed that TE account for 34.94\% of the $B$. oleae genome. In Drosophila, TE genome coverage is variable, ranging from $2.7 \%$ in $D$. simulans to $24.9 \%$ in D. ananassae [71] and is highly correlated with genome size [72]. In the more closely related species, $C$. capitata, TE constitute $18 \%$ of the genome [44]. In terms of genome coverage, Class II DNA transposons accounted for $16.15 \%$ of the genome while Class I retrotransposons accounted for $10 \%$ of the genome. We attempted to annotate the $B$. oleae TE down to superfamily level using TEannot (Supplementary Table S8) but only $5 \%$ of the genome was annotated. Nevertheless, among the annotated families, Tc1-mariner were the most numerous with 1.8 million copies. The Tc1-mariner are ubiquitous Class II TE that form the largest group of eukaryotic TEs [73]. In insects the Tc1-mariner superfamily shows the highest level of horizontal transfer [74]. Class II TE and particularly Tc1-mariner and Piggy$B a c$ TE are of huge significance in Tephritidae sterile insect technique (SIT) as they have been used in medfly control and could be useful in $B$. oleae control $[75,76]$.

\section{Functional genome annotation and curation}

We performed extensive RNA sequencing of the olive fruit fly. RNA was extracted from 12 tissues and/or stages; 6 from female, 1 from male and 5 of mixed origin. The tissues and/or organs included eggs, larvae, pupae, heads, testes among others (Supplementary Table S9). RNA-seq data was collected from these tissues and stages since they were used to address other important questions of the $B$. oleae biology, such as the reproductive and the olfactory system [28, 29]. Between 29 and 55 million reads per sample were generated and used to perform de novo transcript assembly using Trinity [77]. This produced 133,003 transcripts with a median transcript length of $503 \mathrm{bp}$ (Supplementary Table S10 and Supplementary Figure S11). The completeness of the assembly was evaluated by querying Arthropoda, Insecta, and Diptera Basic Universal Single Copy Orthologs (BUSCOs) in the assembly of which 99, 98.4, and 94.8\% are present as complete (Supplementary Table S11) suggesting that the transcriptome captured most genes. Overall alignment rates of RNA-seq data ranged from 88 to 94\% (Supplementary Figure S8).

A more comprehensive protein coding gene-prediction pipeline, JAMg [78], was used to derive a more complete transcriptome of the olive fruit fly, integrating the RNAseq datasets as a source of evidence. This pipeline has previously been used to annotate other Tephritidae genomes with good comparison to NCBI eukaryotic annotation pipeline [44]. The JAMg derived official gene set (OGS) contains a total of 16,455 protein-coding genes. Further, 3920 genes $(23.8 \%)$ are predicted to have variants (isoforms) giving a total of 25,885 isoforms. Excluding isoforms, the mean gene (exons and introns) and transcript (coding and non-coding exons only) length is $11,545 \mathrm{bp}$ and $2109 \mathrm{bp}$, respectively, with the longest gene found to be $299,321 \mathrm{bp}$ and the longest transcript being $61,439 \mathrm{bp}$. The top BLAST hit for the longest gene was fruitless which encompasses $131 \mathrm{~kb}$ genomic region in D. melanogaster [79] while the longest transcript was the $8 \mathrm{~kb} D$. melanogaster beta-spec.

To determine the completeness of the JAMg transcriptome, Diptera BUSCOs were searched. Of the 2799 BUSCOs 2703 (96.57\%) were captured. This is comparable to 99.3 and $99.4 \%$ identified in D. melanogaster and C. capitata, respectively (see Supplementary Figure S12 for comparison to 18 other insect proteomes). Alignment of RNA-seq data derived from 12 different tissues showed alignment rates from 64 to $77 \%$ (Supplementary Figure S8). Further, 55\% of all predicted genes could be assigned to chromosomes while $45 \%$ were located on scaffolds/contigs that are not yet assigned to individual chromosomes (Supplementary Figure S13). Each B. oleae 
protein (or the longest protein for multi-isoform genes) was BLAST-searched against the Swiss-Prot database (Evalue of 0.0004). Out of the 16,455 genes, 10,505 (64\%) had significant hits. Blast2GO [80] was used to retrieve domain and motif signatures via Interproscan [81] analysis followed by identification of gene ontology (GO) terms via mapping and assignment of GO terms to sequences through functional annotation. Except for 51, all proteins with BLAST hits could be mapped and annotated. The top GO terms in each of Biological function, Cellular function, and Molecular function categories are shown in Supplementary Figure S14.

The $B$. oleae mitochondrial genome (GenBank accession NC_005333.1) has been previously described [82] This $15.8 \mathrm{~kb}$ genome encodes 13 protein coding genes/subunits (NADH dehydrogenase, cytochrome b and c, ATP synthase), 22 tRNA genes and 2 rRNA genes (12S and 16S).

\section{Orthology and phylogeny relationship to other insects}

Using complete proteomes, we analyzed phylogeny relationships between $B$. oleae and 18 other insects, 15 of which were previously analyzed but the authors used selected orthologs [44]. Traditionally, evolutionary relationships are inferred from multiple sequence alignment of selected homologous proteins. However, alignmentfree methods which make use of whole proteomes rather than selected proteins have been shown to perform comparably [83]. We used Prot-SpaM [83] to infer pairwise distances of the 19 species. A phylogenetic tree
(Fig. 4) was estimated using Neighbor-Joining algorithm [84] implemented in T-REX [85] and viewed using iTOL [86]. This un-rooted phenetic tree largely recapitulates the previously reported evolutionary tree [44] showing that $B$. oleae is more closely related to the other tephritid Bactrocera dorsalis, Zeugodacus cucurbitae, and C. capitata and more distantly related to D. melanogaster. The other insects were also well clustered according to their order or suborder.

Orthologs among the 6 most closely related insects; $D$. melanogaster, M. domestica, C. capitata, Z. cucurbitae, $B$. dorsalis, and B. oleae were identified using OrthoFinder [87]. A total of 12,413 orthogroups were generated (Table 3, Supplementary Table S12). Out of a total of 144,022 total protein sequences $90 \%$ were assigned to an orthogroup. D. melanogaster and B. oleae had the highest number of proteins not assigned to an orthogroup; 16.6 and $16 \%$, respectively. A total of 1395 orthogroups were identified that contain a single protein from each of the 6 species and another 7286 orthogroups that had one or more' protein from each species. As it would be expected, B. oleae shared more orthogroups with $C$. capitata than with $D$. melanogaster or $M$. domestica (Fig. 5, see Supplementary Figure S15 for a comparison of all 6 species).

\section{Identification of developmental stage-specific genes}

The olive fruit fly is a holometabolous insect. Egg development lasts $66-70 \mathrm{~h}$ in $B$. oleae but this is linearly

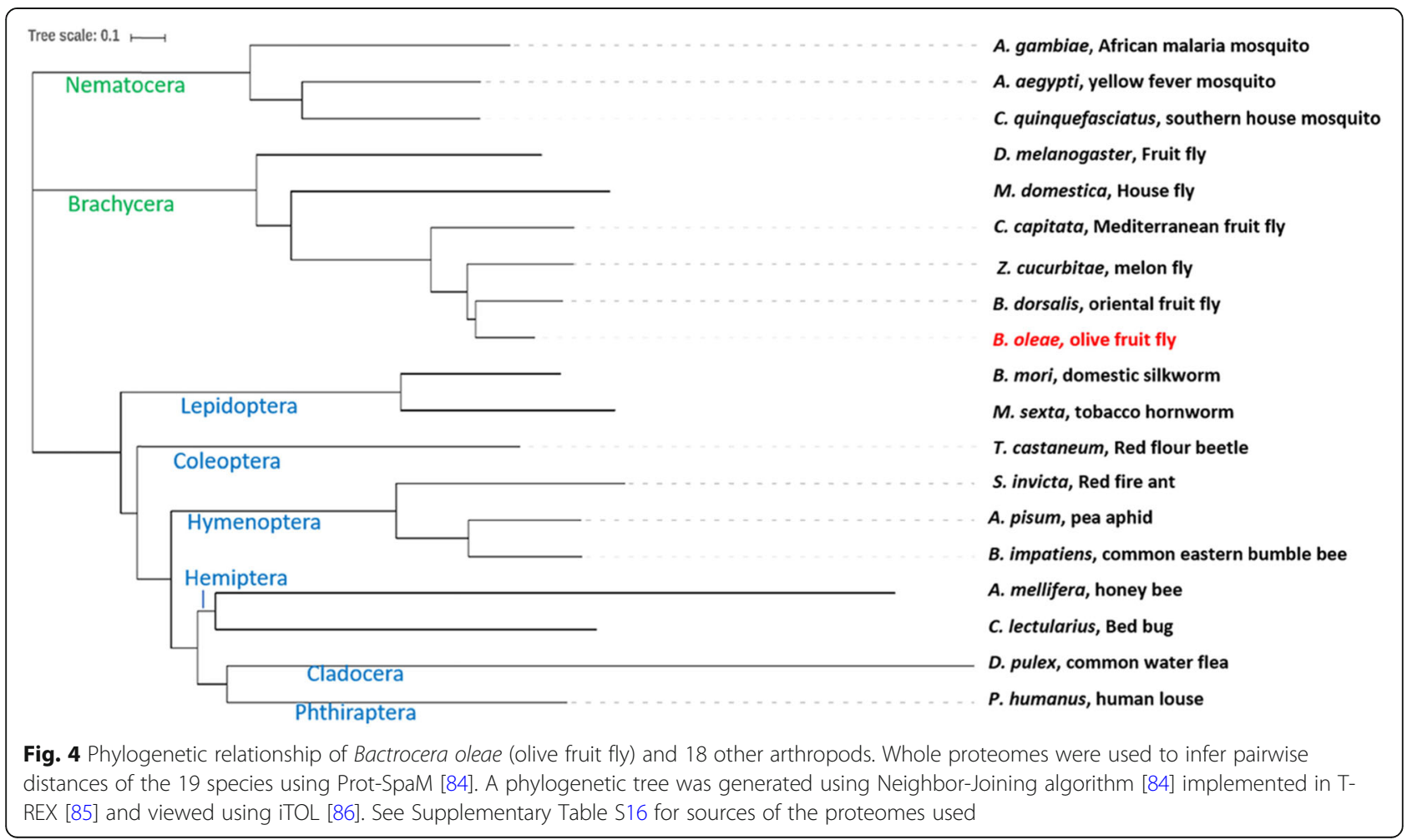


Table 3 Summary of orthologous proteins among six most closely related Dipteran insects

\begin{tabular}{|c|c|c|c|c|c|c|}
\hline & B. dorsalis & B. oleae & C. capitata & D. melanogaster & M. domestica & Z. cucurbitae \\
\hline Total proteins & 20,833 & 25,885 & 22,949 & 30,588 & 19,552 & 24,215 \\
\hline Proteins in orthogroups & 20,296 & 21,745 & 22,135 & 25,523 & 16,931 & 23,477 \\
\hline Unassigned proteins & 537 & 4140 & 814 & 5065 & 2621 & 738 \\
\hline Proteins in orthogroups (\%) & 97.4 & 84 & 96.5 & 83.4 & 86.6 & 97 \\
\hline Unassigned proteins (\%) & 2.3 & 16 & 3.5 & 16.6 & 13.4 & 3 \\
\hline Orthogroups containing species & 11,417 & 10,991 & 11,294 & 10,274 & 10,304 & 11,521 \\
\hline Species-specific orthogroups & 2 & 17 & 12 & 113 & 30 & 5 \\
\hline Proteins in species-specific orthogroups (\%) & 6 & 90 & 71 & 490 & 131 & 23 \\
\hline Proteins in species-specific orthogroups (\%) & 0 & 0.3 & 0.3 & 1.6 & 0.7 & 0.1 \\
\hline
\end{tabular}

Bactrocera dorsalis, Bactrocera oleae, Ceratitis capitata, Drosophila melanogaster, Musca domestica, and Zeugodacus cucurbitae. Orthologous proteins were identified and grouped using OrthoFinder [87]. \% = percentage, See Supplementary Table S16 for the source of proteins used

dependent on temperature $[89,90]$. The eggs included in the current study were pooled over $24 \mathrm{~h}$. At this stage the embryos have undergone the maternal-to-zygotic transition, completed blastoderm formation and are just about starting gastrulation [89]. The larva is a specialized feeding stage and the most destructive stage to the olives. The larvae accumulate mass over 10-14 days and undergo successive rounds of molting where the old cuticle is shed and a new one built in response to a hormone [91] called ecdysone [92]. The 28 larvae used in the current study were pooled over the 3 instar stages although 20 were from Instar 1 . The $8-12$ days immobile pupa stage is a molecularly controlled and highly dynamic stage where the larval tissues are broken down by apoptosis [93] and adult tissues such as wings emerge. The genes specific to the individual stages in the olive fruit fly have not been elucidated. Here, we picked the top 1100 most variable genes across the four metamorphic stages and performed a principal component analysis (PCA, Fig. 6). The first principal component ( $\mathrm{PC} 1$, accounting for $38 \%$ of the total variation), contrasted the adult stage from the egg, larva, and pupa stages. PC2 (accounting for 33\% of the total variation), separated the pupae from the egg and larvae. The first principal component thus captures the huge transcriptional differences between the adult and the other developmental stages. The egg and larvae were coseparated perhaps due to most of our larvae coming from L1 stage. To determine the genes that account for these differences we plotted the 'circle of correlations' which suggested highly correlated and exclusive sets of genes expressed at the different developmental stages (black dots in Fig. 6). Indeed, hierarchical clustering showed clear clusters of genes that were only highly expressed at specific stages (Supplementary Figure S16). Temporal gene expression has been suggested to follow a Gaussian distribution [95]. In order to identify the developmental stage-specific genes, we clustered all expressed genes using Dirichlet process Gaussian process (DPGP) [96] which jointly models data clusters with a Dirichlet

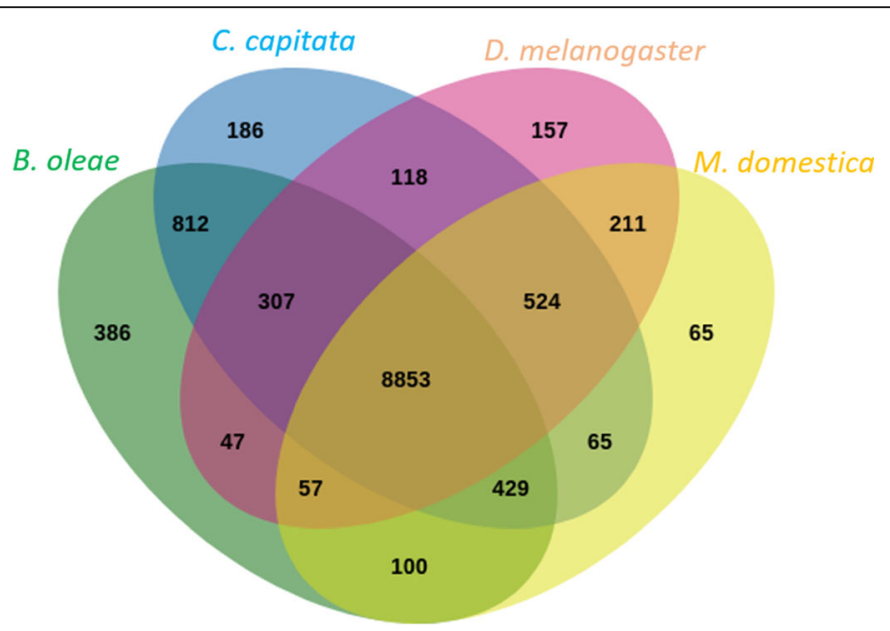

Fig. 5 Venn diagram of shared orthogroups among B. oleae, C. capitata, D. melanogaster, and M. domestica. Orthologous proteins were identified and grouped using OrthoFinder [87]. Shared and unique orthogroups are plotted using Jvenn [88]. See Supplementary Table S16 for sources of the proteomes used 


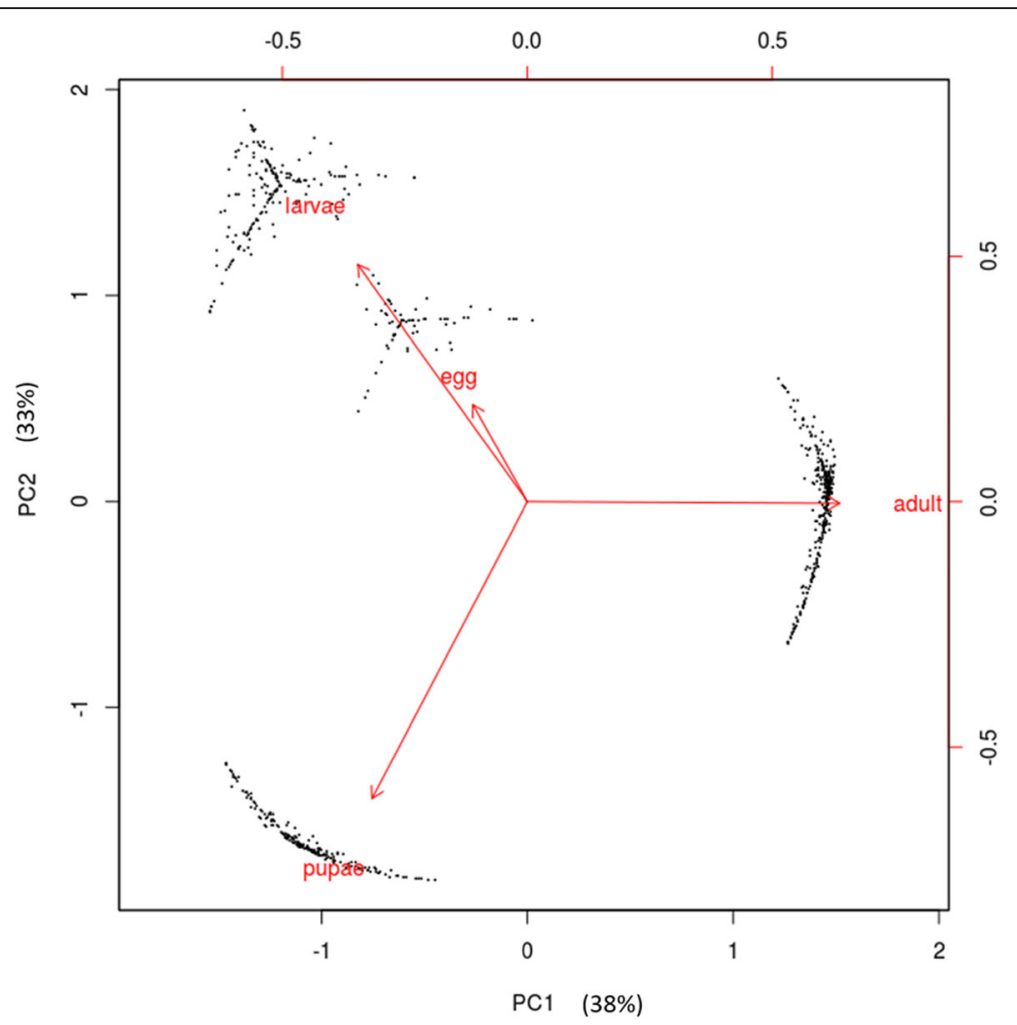

Fig. 6 Principle component analysis (PCA) of 1100 most variable genes among the 4 metamorphotic stages. Gene expression (transcripts per million) was calculated for each of the stages; egg, larvae, pupae, and adult using RSEM [94]. A coefficient of variation was determined for each gene and used to determine the most variable genes. Eigenvector coordinates for the stages (egg, larvae, pupae, and adult) on the first 2 components are shown in red. Coordinates of the individual genes on the first 2 principle components (circle of correlation) are shown as black dots

process and temporal dependencies with Gaussian processes. From this, we identified 7086 genes whose expression peaks at different stages, suggesting specific roles for these genes during defined developmental periods (Fig. 7, see Supplementary Table S13 for the genes and corresponding D. melanogaster BLAST hits).

Enrichment analysis was performed on the genes in each category using gProfiler [97] and ranked by the adjusted $p$-value. The most enriched GO terms in the egg (Supplementary Figure S17A) were metabolic, biosynthesis, and developmental processes. The most enriched GO terms in the larvae (Supplementary Figure S17B) were chitin metabolic processes, cuticle development, body morphogenesis. For the pupae stage, the most enriched processes were development and morphogenesis related; e.g. multicellular organism development, tube development, imaginal disc morphogenesis, eye development (Supplementary Figure S17 C). We provide a list of the enriched processes in each stage in Supplementary Table S14.

\section{Discussion}

We have assembled the whole genome of Bactrocera oleae using both short-read (paired-end and mate-pair approaches), long-read, and linked-reads technologies which encompass all the currently available next generation sequencing technologies. The linked-reads approach generated the most contiguous assembly with a scaffold N50 of $2.16 \mathrm{Mb}$. This was then chosen as a backbone for scaffolding and gap-closing, which more than doubled the genome contiguity; N50 increased to $4.69 \mathrm{Mb}$. The final assembly (GenBank accession GCA_ 001188975.4) is one of the most highly contiguous Tephritidae assembly in the NCBI catalogue (see Supplementary Figure S18 for some comparisons). We were able to achieve this because the laboratory strain of the olive fruit fly used for genome sequencing has low heterozygosity due to (i) low level of natural polymorphism, (ii) significant bottleneck during colonization, and (iii) long period of laboratory rearing (over 45 years) without any admixture. This significantly reduces the ambiguities during the assembly process thus increasing contiguity. Further, we were able to extract high molecular weight DNA that was used to prepare long-read and linked-read sequencing libraries. Due to technological advancements, the library preparation and sequencing costs of short- and long-read technologies is converging at USD 20-40 per Gb [98]. Linked-reads library 

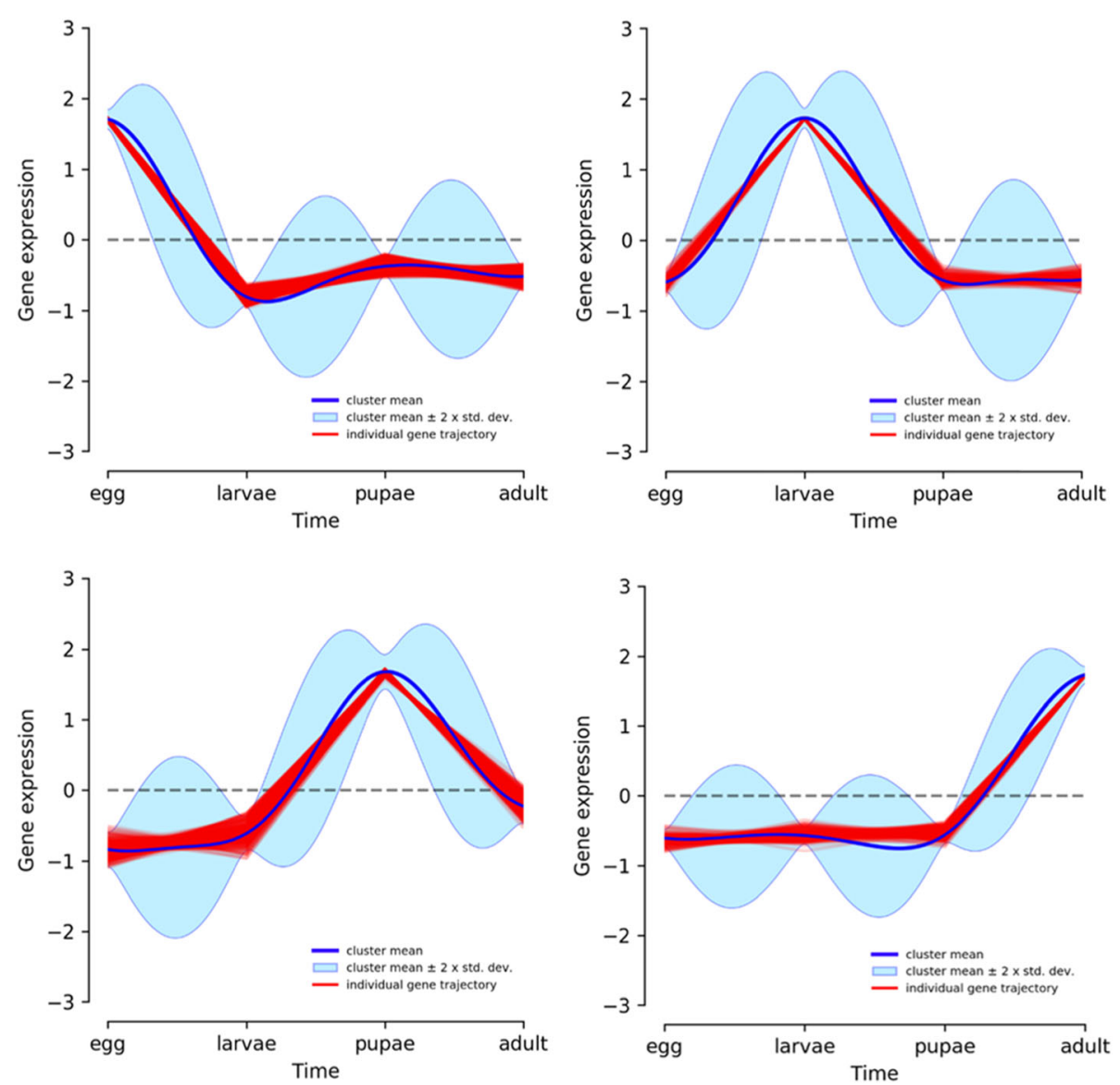

Fig. 7 Drichlet process Gaussian process (DPGP) [92] modeling and clustering of gene expression. Gene expression (transcripts per million) was calculated for each of the 4 metamorphotic stages; egg, larvae, pupae, and adult using RSEM [94] and the expression matrix used to determine genes that only peak at the corresponding stage

preparation adds another USD 475 per sample. Considering large genome sequencing efforts like i5k, we find that de novo genome assembly using the linkedreads approach followed by scaffolding and gap-closing using long-reads provides high quality assemblies. For small organisms, like flies, that would be difficult to yield micrograms of high-quality DNA from a single organism in order to reduce heterozygosity and thus increase contiguity and accuracy during genome assembly the linked-reads approach is highly suited. The Hi-C library preparation approach [99] has been shown to yield chromosomal length assemblies, but the method relies on the existence of suitable high-quality tissue and a contiguous assembly as input (ideally with an N50 of $1 \mathrm{Mb}$ as for example in the case of applying the Dovetail $^{\text {Ts }} \mathrm{Hi}-\mathrm{C}$ and HiRise ${ }^{\text {Tw }}$ methodology [100]). The Hi-C method and its variant, the 'Chicago' method [101], which only requires high molecular weight DNA, can be used whenever possible to increase contiguity $[100,102]$.

The assembly presented here will enormously boost the understanding of the olive fruit fly's biology and genome evolution and shed light to its particularities. For example, as a strictly monophagous insect, olive fruit fly larvae restrict their diet to the olive sap, adapting their physiology to a single nutritional resource and relying on intestinal symbionts to supply essential dietary components that are not supplied by the olive fruit $[103,104]$. Being consummate specialists, olive fruit fly larvae may restrict and at the same time specialize their defenses to the plant host, the olive fruit. Such adaptations inevitably should be reflected in its genome and these can now be investigated (for review on insect genome adaptation to host plants see $[105,106])$.

Dipteran flies typically have 6 diploid chromosomes with an XY heterogametic system [107]. Although the X chromosome largely retains the organization of its autosome ancestor, the $\mathrm{Y}$ chromosome undergoes massive gene decay and general degeneration accompanied by accumulation of repetitive sequences [108]. The highly repetitive nature of the $\mathrm{Y}$ chromosome makes it the most challenging to assemble in genome sequencing efforts. The Y chromosome is not devoid of genes. 
Indeed, in D. melanogaster, the $\mathrm{Y}$ chromosome contains at least 12 genes [35]. However, some of these genes contain megabase size introns and repeats making them difficult to sequence which then necessitates the use of long-read approaches to assemble them [109, 110]. All D. melanogaster $\mathrm{Y}$ genes have male-specific functions yet only 3 seem to be conserved while 7 were acquired less than $63 \mathrm{Myr}$ ago showing the high rate of gene gain in flies [35]. These factors demonstrate the need to assemble the highly dynamic but critical Y chromosome and identify its genes. Methods of $Y$ chromosome identification include PCR amplification of Y-linked markers as was done for Anopheles gambiae [111], bacterial artificial chromosome cloning followed by mapping and sequencing as was done for humans and primates [112] or BLAST search of unmapped scaffolds in assembled genomes as was done for D. melanogaster [113]. Newer technologies include BioNano maps [114], Hi-C [115] and fluorescence in situ hybridization (FISH) of markers. Another method uses flow cytometry sorting to enrich for the Y chromosome followed by short and long read sequencing and RNA-seq [116]. All these methods have challenges in cost or applicability. Here, we adopted the chromosome quotient method that has been used to successfully identify Y-chromosome sequence in Anopheles stephensi and A. gambiae [49]. We used our extensive dataset including female WGS, male WGS, and our contiguous male genome assembly to apply the Chromosome Quotient method and identify putative $\mathrm{Y}$ and $\mathrm{X}$ chromosome scaffolds. Indeed, we assembled $3.9 \mathrm{Mb}$ of B. oleae $\mathrm{Y}$ chromosome and $6 \mathrm{Mb}$ of $\mathrm{X}$ chromosome. We used sequences from these putative $Y$ chromosome scaffolds to design PCR primers and thus experimentally confirmed the amplification of male-specific fragments. The sequence of these scaffolds amounts to $1.7 \mathrm{Mb}$ and provides a valuable resource for $\mathrm{Y}$ chromosome gene identification. Previously, $700 \mathrm{~kb}$ of Y chromosome was identified for Bactrocera tyroni using genotype-bysequencing data and whole-genome resequencing [117]. $\mathrm{Y}$ chromosomes, however, remain difficult to assemble. The D. melanogaster Y chromosome is estimated at 40 $\mathrm{Mb}$ but only $4.2 \mathrm{Mb}$ is assembled into contigs/scaffolds (Flybase release r6.28), and only recently an improved assembly yielded a total of $14.6 \mathrm{Mb}$ of Y-linked sequence [118]. Importantly, the B. oleae Y chromosome is home to the male sex determining factor that is responsible for initiating the sexual determination molecular cascade in Tephritidae and had remained elusive thus far. The male sex determining factor, $M o Y$ (maleness on the $\mathrm{Y}$ ) has recently been discovered in Ceratitis capitata and Bactrocera oleae [38], and indeed, the gene is well captured in our PCR confirmed Y chromosome assembly (scaffold LGAM02015747 in GCA_001188975.4 assembly). Work is underway to identify other genes on $B$. oleae $\mathrm{Y}$ chromosome which will provide critical information to our understanding of Tephritidae Y chromosome evolution and factors contributing to the male phenotype.

\section{Methods \\ Breeding of the insects}

The olive fruit fly, Bactrocera oleae, 'Demokritos' strain, that is considered in this study was originally sourced from the Nuclear Research Centre in Athens, Greece where it has been maintained for over 45 years. We have maintained this strain in our laboratory for over 15 years with no wild flies added since then, hence the strain has maintained a genetic uniformity. Olive flies were reared in appropriate holding cages at $25 \pm 1{ }^{\circ} \mathrm{C}, 60 \pm 10 \%$ relative humidity and $14 \mathrm{~L}: 10 \mathrm{D}$ cycles according to the conditions described in [119]. B. oleae individuals were immediately sexed upon their emergence and separated until DNA extraction.

\section{Genomic DNA preparation}

High molecular weight (HMW) B. oleae genomic DNA was extracted separately from virgin male and female adult flies following the 'nuclei DNA extraction' procedure described in Zhang et al. [120]. Generally, whole body insects were frozen in liquid nitrogen and ground in a mortar with pestle into fine powder. The DNA was extracted with phenol/chloroform, precipitated with ethanol and resuspended in TE buffer $(10 \mathrm{mM}$ Tris- $\mathrm{HCl}$, 1 mM EDTA, pH 8).

\section{Library preparation}

To generate the paired-end libraries, Illumina TruSeq DNA library preparation kit was followed together with genomic DNA extracted from both male and female flies. The DNA was sequenced using Illumina HiSeq sequencers. The mate-pair libraries were prepared following the Nextera mate-pair library preparation kit from Illumina according to manufacturer instructions. The target sizes for the libraries were: $3 \mathrm{~kb}, 5 \mathrm{~kb}$, and 10 kb. Pacific Biosciences Inc. (PacBio, California, USA) DNA libraries were prepared following the $20 \mathrm{~kb}$ Template Preparation protocol and SMRTbell Template Prep Kit 1.0 using $7.5 \mu \mathrm{g}$ of DNA and then sequenced on the RSII sequencer. Oxford Nanopore genomic library preparation protocols SQK-MAP 006, SQKNSK007, and SQK-LSK108 were followed using $5 \mu \mathrm{g}$ of HMW DNA. DNA was sequenced on the MinION. To generate the linked-reads libraries, HMW DNA was sent to 10x Genomics (CA, USA) where the library preparation was performed. Final library size was sequenced on one lane of an Illumina HiSeq XTen sequencer as 150 bp paired-end generating a total of 414 million reads or 125 Gb. The mate-pair, PacBio, ONT, and linked-reads 
libraries were prepared using genomic DNA extracted from male insects only.

\section{RNA extraction and sequencing}

A total of 12 RNA samples were collected from different developmental stages and tissues (Supplementary Table S9). The sample sources included adult flies, embryos (eggs), larvae, pupae as well separate tissues including heads, legs, ovipositor, testes, sex organs, and thorax. Total RNA was extracted using the Trizol method and quality-checked as previously described [28]. The TruSeq stranded library preparation protocol (Illumina) was followed by $100 \mathrm{bp}$ paired-end sequencing with Hiseq 2000/2500 sequencers (Illumina).

\section{Genome size and heterozygosity estimation}

The frequency of k-mers of length $=23$ bases was calculated using BBMAP [57] followed by genome size estimation using GenomeScope [58].

\section{Genome assembly}

The initial assembly was generated using both male and female paired-end reads. The reads were combined and assembled together using a short-read assembler Ray [41] which was run with a range of kmer values (see Supplementary Table S1). This short-read assembly was then scaffolded with three mate-pair libraries (see Supplementary Table S1) using SSPACE (version 3.0) [42]. As a final step, PacBio reads were used to fill the sequence gaps left behind by the scaffolding process. This assembly was filtered for scaffolds with more than $10 \mathrm{X}$ of average Illumina coverage and a minimum length of $500 \mathrm{bp}$ and submitted to NCBI (GenBank assembly accession: GCA_001188975.2). To generate an ONT based assembly, ONT sequence reads were used for de novo genome assembly of the olive fly using Canu [121]. This assembly was named ONT-only. To assemble a hybrid long-read assembly, reads generated from ONT sequencing for the olive fly and those generated from PacBio sequencing were combined and used to generate a separate hybrid assembly (named ONT-PacBio) using Canu (version 1.5).

\section{Optimization and de novo genome assembly using linked-reads}

The Supernova assembler was used to develop the de novo linked-reads assembly. Several rounds of optimization were performed by changing the number of partitions and coverage required to give the most contiguous assembly (as measured by the assembly NG50, assuming a genome size of $320 \mathrm{Mb}$ [23]). We finally selected the genome assembly with $74 \mathrm{X}$ coverage and 540,000 barcodes per partition. The resulting genome was analyzed using Quast [43] using default parameters. This genome was named 10x-only.

\section{Assembly polishing with Pilon}

The 387 million Illumina paired-end sequencing reads (yielding $\sim 100 \mathrm{X}$ coverage of the olive fly genome) derived from the 10x Genomics experiment was used to correct all assemblies. Reads were aligned to the genomes using BWA-MEM and resulting alignment files processed using Pilon [122]. The polishing was performed in two rounds to derive the polished assemblies. Pilon was run with default parameters. Due to inherent errors in long-read derived genome assemblies, the uncorrected and error-corrected versions of these assemblies were aligned to the assembly derived from 10x Genomics data, using MUMmer [123] (version 3.23) to determine alignment identity. The parameters used were "-1 100 -c 500 -maxmatch". The '.delta' output was analyzed with dnadiff (part of MUMmer software) to determine the average alignment identity.

\section{Identification of symbiont derived sequences}

In the first approach, we mapped raw reads (SRX5578411 and SRX5557611) and the male genome assembly (GCA_ 001188975.4) of the olive fruit fly to reference genomes of Wolbachia, Spiroplasma, and Cardinium using MIRA v4.0 and bowtie2. For the Wolbachia mapping exercise we used complete and draft genomes that were publicly available (4688 contigs in total) as reference sequences. For the Spiroplasma mapping we used the following complete genomes: (a) Spiroplasma chrysopicola DF-1, (b) Spiroplasma syrphidicola EA-1, complete genome, (c) Spiroplasma taiwanense CT-1, complete genome, (d) Spiroplasma diminutum CUAS-1. For Cardinium, the Cardinium endosymbiont cEper1 of Encarsia pergandiella was used as a reference genome. In the second approach, we downloaded 235,684 complete and draft genomes that have been deposited to NCBI (June 2019). These sequences were used as a custom BLAST database in order to identify bacterial sequences that have been filtered into the assembly of the $B$. oleae genome. Blast results were visualized using BLASTGrabber v.2.

\section{Benchmarking universal single-copy Orthologs (BUSCOs) analysis}

Assembly completeness (genome or transcriptome) was assessed by querying the presence of orthologous sets of evolutionarily conserved genes termed Benchmarking universal single-copy orthologs (BUSCOs) [56] from 4 different phylogenetic lineages; Eukaryota, Arthropoda, Insecta, and Diptera. First, datasets for the 4 different lineages were downloaded from busco.ezlab.org. The assemblies were then successively queried for the presence of each lineage specific BUSCO using the BUSCO software (version 2.0.1). 


\section{$\mathrm{Y}$ and $\mathrm{X}$ chromosome identification}

In order to find putative $\mathrm{X}$ or $\mathrm{Y}$ chromosome scaffolds we used the Chromosome Quotient method [49] which calculates the median ratio of female to male coverage for each scaffold. The resulting quotient values will cluster around zero, one or two for $\mathrm{Y}$, autosome or $\mathrm{X}$ scaffolds respectively. Before aligning the reads, repeats are masked from the assembly using RepeatMasker. We aligned 40x coverage of male and female reads to a hard-masked version of the assembly and for each set, we calculated the depth at each base for all scaffolds. We further filtered out positions with less than 10x of male coverage to ensure a minimum of evidence from male DNA.

\section{Validation of Y-chromosome specific scaffolds}

Putative Y-derived scaffolds were validated through standard PCR and real-time quantitative PCR (RT-qPCR). Specifically, DNA was extracted from three pools of virgin male and female insects each one containing 10 insects. Eighty-five pairs of primers were designed using the Primer3 [124]. PCR reaction was carried out in a final volume of $20 \mu \mathrm{l}$, using $1.5 \mathrm{mM} \mathrm{MgCl} 2,1 \mathrm{x}$ PCR reaction buffer, 1 Unit Taq DNA polymerase (Bioline, London, UK), 0.35 pmol of each forward and reverse primers and $0.8 \mathrm{mM}$ dNTPs. The amplification conditions were as follows: $94{ }^{\circ} \mathrm{C} 4 \mathrm{~min} ; 94^{\circ} \mathrm{C} 30 \mathrm{~s}, 55^{\circ} \mathrm{C} 30 \mathrm{~s}, 72{ }^{\circ} \mathrm{C} 2 \mathrm{~min}$ for 30 cycles; $72^{\circ} \mathrm{C} 5 \mathrm{~min}$. PCR products were identified by $1-$ $1,5 \%$ agarose gel electrophoresis. RT-qPCR was carried out in a final volume of $15 \mu \mathrm{l}$, using $1 \mu \mathrm{l}$ from a 1:10 dilution of the cDNA template, 2X SYBR Select Master Mix (Applied Biosystem) and $300 \mathrm{nM}$ of each primer. The amplification conditions were: polymerase activation at $50^{\circ} \mathrm{C}$ for $2 \mathrm{~min}$, DNA denaturation step at $95^{\circ} \mathrm{C}$ for $4 \mathrm{~min}$, followed by 50 cycles of denaturation at $95^{\circ} \mathrm{C}$ for $10 \mathrm{~s}$, annealing/extension and plate-read at $55^{\circ} \mathrm{C}$ for $20 \mathrm{~s}$ and finally, a step of melting curve analysis at a gradual increase of temperature over the range $55^{\circ} \mathrm{C}$ to $95^{\circ} \mathrm{C}$. The reactions were carried out on a Bio-Rad Real-time thermal cycler CFX96 (Bio-Rad, Hercules, CA, USA) and data were analyzed using the CFX Manager ${ }^{\mathrm{Tw}}$ software. All PCR reactions were performed in triplicate (i.e., three technical replicates).

\section{Cloning of probe sequences for in situ hybridization}

Specific primers were designed using Primer3 to amplify segments of scaffolds for which there was no previous mapping information available. The probe amplification was carried out in a $20 \mu \mathrm{l}$ PCR reaction volume using $1.5 \mathrm{mM} \mathrm{MgCl}_{2}$, $1 \mathrm{X}$ PCR reaction buffer, 1 unit Taq DNA polymerase (Bioline, London, UK), $0.35 \mathrm{pmol}$ of each forward and reverse primers and $0.8 \mathrm{mM}$ dNTPs. The amplification conditions were as follows: $94{ }^{\circ} \mathrm{C} 4$ min; $94{ }^{\circ} \mathrm{C} 30 \mathrm{~s}, \mathrm{Tan}^{*}{ }^{\circ} \mathrm{C} 30 \mathrm{~s}, 72{ }^{\circ} \mathrm{C}$ extension time for
30 cycles; $72^{\circ} \mathrm{C} 5 \mathrm{~min}$. The PCR products after electrophoresis were gel purified by the Wizard ${ }^{\circ} \mathrm{SV}$ Gel and PCR Clean-Up System (Promega, Madison, WI, USA) following the manufacturer's instructions, ligated into TA cloning vector pTZ57R/T (Thermo Scientific InsTAclone PCR Cloning Kit) and finally used to transform electrocompetent $E$. coli $\mathrm{DH} 5 \alpha$ cells according to standard procedures. The recombinant plasmid DNA was finally isolated with the use of the Promega Wizard Plus Minipreps DNA Purification System according to the supplier's instructions.

\section{Chromosome preparations and in-situ hybridization}

Polytene chromosome spread preparations were obtained from the salivary glands of third instar larvae and young pupae (1-2 days old) [125]. The random priming method was used to generate the digoxigenated dUTP (Dig-11dUTP) labelled probes. Hybridization was performed at $62^{\circ} \mathrm{C}$ and signal detection was performed using the DIG DNA Labeling and Detection kit (ROCHE Diagnostics, Mannheim, Germany) according to Drosopoulou et al. [125]. Two to three preparations were hybridized with each probe, and at least ten well spread nuclei per preparation were analyzed. The pretreatment of chromosome preparations, hybridization, detection and image analysis are described in detail in $[125,126]$. The hybridization sites were identified according to the available polytene chromosome maps $[52,54]$.

\section{Transposable element (TE) identification}

We used the PiRATE [67] pipeline for TE identification. Starting with the assembled genome we used the "similarity-based" tools (RepeatMasker [127]; TE-HMMER), "Structural-based" tools (MITE Hunter [128], HelSearch [129], LTR Harvest [130], SINE-Finder [131], MGEScanLTR [132]), and "Repeatitiveness-based" tools (TEdenovo [69], RepeatScout [133]). TEs overlapping by $100 \%$ of a larger element were removed using CD-HIT-est [134] and the remaining TE classified using PASTEC [69]. Following TE library generation, the sequences were BLAST'ed. against the $B$. oleae proteome and best hits with $>50 \%$ alignment identity, $>100$ nucleotide alignment and Evalue $>0.001$ were removed from the TE library. Finally, the library was used to annotate the genome using TEannot [70].

\section{De novo transcriptome assembly}

We used a pipeline developed following the protocol described in Haas et al. [135] and mostly based on the Trinity assembly software suite [77]. Normalization was performed in order to reduce memory requirement and decrease assembly runtime by reducing the number of reads, using the Trinity normalization utility [77] inspired by the Diginorm algorithm [136]. Haas et al. 
[135] showed that normalization results in full-length reconstruction to an extent approaching that based on the entire read set. In addition, each assembly contig and component were analyzed using the Trinotate annotation pipeline. We also performed Trinity genomeguided transcriptome assembly.

\section{Genome feature and functional annotation}

Feature annotation to generate the official B. oleae gene model set (OGS) was completed using the JAMg annotation pipeline [78] as previously applied [44]. Briefly, the pipeline involved repeat masking using RepeatModeler (v1.0.8), RepeatScout (v1.0.5), and RepeatMasker (v4-06), and the generation of transcriptome database for model training using Augustus. A separate prediction was run using GeneMark-ES (4.38). As EvidenceModeler removes the UTR and alternative transcripts predicted from Augustus, we used PASA to update these models and create the final JAMg OGS. Functional annotation of $B$. oleae gene models predicted by the JAMg annotation pipeline was performed using Blast2GO [80] included in OmicsBox version 1.1.78. Each protein (or the longest protein for multi-isoform genes) was Blastsearched again the Swiss-Prot database (Evalue, 1e-4) with output format 15 selected. XML Blastp results and sequences were imported into Blast2GO [80] and used to retrieve domain and motif signatures via Interproscan [81] analysis followed by identification of gene ontology (GO) terms via mapping and assignment of GO terms to sequences through functional annotation.

\section{Phylogenetic classification}

We used Prot-SpaM [83] to infer pairwise distances of 19 species using complete proteomes. A phylogenetic tree was generated using Neighbour-Joining algorithm [84] implemented in T-REX [85] and viewed using iTOL [86].

\section{Identification of orthologous proteins}

Orthologs among $D$. melanogaster, $M$. domestica, $C$. capitata, Z. cucurbitae, B. dorsalis and B. oleae were identified using OrthoFinder [87]. Supplementary Table S12 contains all orthogroups and the proteins from each species that belong to respective orthogroups.

\section{Principle component analysis and hierarchical clustering}

Gene expression (transcripts per million, TPM) was calculated for each of the 4 metamorphotic stages; egg, larvae, pupae, adult using RSEM [94] and used to calculate gene z-score on the log transformed TPM. Principle component analysis on the 1100 topmost variable genes among the stages was performed by the "prcomp" function then plotted by the "biplot" function both of R statistical software.

\section{Temporal clustering of developmental stage-specific} genes

The expression matrix (transcripts per million, TPM) filtered for genes that were not expressed at any of the stages was used as input to Dirichlet process Gaussian process (DPGP) [96] to cluster genes with similar expression profiles. Clusters of Genes in clusters that peak at either of the 4 metamorphotic stages; egg, larvae, pupae, adult were combined and used in gene ontology enrichment analysis using gProfiler [97].

\section{Supplementary information}

Supplementary information accompanies this paper at https://doi.org/10. 1186/s12864-020-6672-3.

\section{Additional file 1. Supplementary materials.}

Additional file 2: Table S1. Sequencing libraries used, sequence results, and kmer optimization for the GCA_001188975.2 assembly. Table S2. Comparison of assembly quality of the 3 main assemblies. Table $\mathbf{S 3}$. Assembly statistics for 9 different assemblies generated. Table S4. Comparison of assembly quality of the $3 Y$ chromosome assemblies. Table S5. Sequences of the primers used for the validation of $Y$ chromosome specific scaffolds. Table S6. Mapping positions and primers used to generate 9 new B. oleae DNA markers in this study. Table S7. Scaffold/contig localization on B. oleae chromosomes. Table S8.

Distribution of the $B$. oleae Transposable elements in the genome assembly. Table S9. Samples and tissues used for the transcriptome sequencing and assembly. Table S10. Summary of the Trinity transcriptome generated from sequencing all the tissues in Supplementary Table S7. Table S11. Assessment of the completeness of the Trinity de novo transcriptome assembly. Table S12. Orthologous genes among 6 closely related insects. Table S13. Genes that peak at different metamorphic stages. Table S14. Gene ontology enrichment analysis for genes that peak at different metamorphic stages. Table S15. Datasets submitted to NCBI and their corresponding accession numbers and description. Table S16. Sources of proteomes and genomes used in Supplementary figures.

Additional file 3: Figure S1. Schematic of the method used to generate the main assembly reported. Figure S2. Genome size and heterozygosity estimation. Figure S3. Contig length at different Nx values for assemblies in Supplementary Table S3. Figure S4. Contiguity plot generated using Quast. Figure S5. Contig length at different Nx values for assemblies in Supplementary Table S4. Figure S6. Plot showing $Y$ chromosome scaffolds/contigs identified in 3 different assemblies (Supplementary Table S4). Figure S7. Total length of scaffolds that were localized to each polytene chromosome and XY chromosomes. Figure S8. Alignment rates of RNA-seq reads from 12 different Bactrocera oleae datasets (see Supplementary Table S9). Figure S9. Complete Basic Universal Single Copy Orthologs (BUSCOs) identified in genome assemblies (Supplementary Table S3). Figure S10. Schematic of the PIRATE pipeline. Figure S11. Histogram of transcripts read lengths. Figure S12. Percentage of Arthropoda Basic Universal Single Copy Orthologs (BUSCOS) captured in 19 arthropod transcriptomes. Figure S13. Number of JAMg predicted B. oleae genes located on the scaffolds assigned to polytene element. Figure S14. Gene ontology (GO) classification of B. oleae JAMg predicted proteins. Figure S15. Detailed orthogroup distribution. Figure S16. Hierarchical clustering of 1100 most variable genes among the 4 metamorphotic stages. Figure S17. Most significantly enriched gene ontology (GO) terms among genes that only peak during development. Figure S18. Contig length at different Nx values for assemblies of selected insects.

\section{Abbreviations}

SIT: Sterile Insect Technique; ONT: Oxford Nanopore Technologies; BUSCO: Basic Universal Single Copy Orthologs; TGS: Third generation Sequencing; PacBio: Pacific Bioscience; ISPRA: Italian National Institute for 
Environmental Protection and Research; kb: kilobase(s); Mb: Megabase(s); Gb: Gigabases; ng: nanogram(s); NCBI: National Center of Biotechnology Information; Myr: Million years; PCR: Polymerase chain reaction; vs: versus; N50: Scaffold/contig length at which $50 \%$ of the total genome length is contained in scaffolds/contigs of that size or longer when all scaffolds/ contigs are ordered from longest to shortest; NG50: N50 except the genome size is fixed and not dependent on sum of scaffolds/contigs; L50: Total number of scaffolds/contigs needed to reach N50; LG50: Total number of scaffolds/contigs needed to reach NG50; rpm: rounds per million; ml: milliliter; $\mu \mathrm{g}$ : microgram; mM: millimolar; EDTA: Ethylene di-amine tetraacetic acid; nM: nanomolar; pM: picomolar; $\mu$ l: microlitre; HMW: High molecular weight; OGS: Official gene set

\section{Acknowledgements}

We would like to thank Louis Letourneau (Genome Quebec, Montreal, Canada) for the original transcript assembly work. This work also benefited from discussions during Coordination Research Projects (CRP) supported by the Joint FAO/IAEA Division of Nuclear Techniques in Food and Agriculture.

\section{Authors' contributions}

$A B$ performed library preparation, sequencing, data analysis, and co-wrote the manuscript. HD performed data analysis, generated genome assemblies, and co-wrote the manuscript. SO performed long-read library preparation and data analysis. JR designed the study, supervised the genomic work and data analysis, co-wrote the manuscript. KTT, M-EG and ES were involved in olive fruit fly tissue isolation. CG and DMC were responsible for linked-read library preparation. KD advised on genome assembly approaches. KTT and MEG were involved in validation of $Y$ scaffolds and manuscript preparation. $K T T, E D$ and PM-T were involved in chromosomal assignment of scaffolds. KDM designed the study, supervised the $Y$ validation and chromosomal assignment of scaffolds, co-wrote the manuscript. AP performed the JAMg annotations and WebApollo updating of data. GT and KB performed the symbiont analysis. All authors reviewed and approved the final version of the manuscript.

\section{Funding}

The genome and transcriptome sequencing were supported by a Stavros Niarchos Fulbright Greek Diaspora Scholarship, Genome Canada Genomics Technology Platform grant, the Canada Foundation for Innovation (CFI) and the CFI Leaders Opportunity Fund (32557), Compute Canada Resource Allocation Project (WST-164-AB) and Genome Innovation Node (244819) to $J R$. AB is a Queen Elizabeth II PhD scholarship recipient. Part of the sequencing cost of this research was also supported by the "ARISTEIA" (MIS524938) Action of the "Operational programme Education and Lifelong Learning", co-funded by the European Social Fund (ESF) and Hellenic National Resources. Further support was provided by the two postgraduate programs of the Department of Biochemistry and Biotechnology of the University of Thessaly ("Biotechnology - Quality Assessment in Nutrition and the Environment" and "Applications of Molecular Biology -Genetics -Diagnostic Biomarkers"). KTT was funded through a postdoctoral studies scholarship from the Hellenic State Scholarship Foundation (IKY); this research is co-financed by Greece and the European Union (European Social Fund-ESF) through the Operational Programme «Human Resources Development, Education and Lifelong Learning» in the context of the project "Reinforcement of Postdoctoral Researchers" (MIS-5001552), implemented by the State Scholarships Foundation (IKY). The NIH Intramural Research Program, National Library of Medicine funded the NCBI Gnomon annotation and the USDA-National Agricultural Library (NAL) provided support for the WebApollo curation website.

\section{Availability of data and materials}

The Genome sequence has been submitted to NCBI with GenBank accession number GCA_001188975.4. All raw reads and RNA-seq data have been submitted to SRA using the study number PRJNA288990. See Supplementary Table S15 for SRA accession numbers for each dataset.

\section{Ethics approval and consent to participate}

Not applicable.

\section{Consent for publication}

Not applicable.

\section{Competing interests}

$J R$ is a member of the MinION Access Program (MAP) and has received freeof-charge flow cells and sequencing kits from Oxford Nanopore Technologies for other projects. JR has had no other financial support from ONT. AB has received re-imbursement for travel costs associated with attending the Nanopore Community meeting 2018, a meeting organized by Oxford Nanopore Technologies. KG and DMC held positions as employees with 10x Genomics (Pleasanton, California, USA).

\section{Author details}

${ }^{1}$ McGill University and Genome Quebec Innovation Centre, Department of Human Genetics, McGill University, Montreal, Canada. ${ }^{2}$ Department of Biochemistry and Biotechnology, University of Thessaly, Biopolis, 41500 Larissa, Greece. ${ }^{3}$ Department of Biology, Aristotle University of Thessaloniki, Thessaloniki, Greece. ${ }^{4}$ Integrated DNA Technologies, Inc., 1710 Commercial Park, Coralville, lowa 52241, USA. ${ }^{5}$ Department of Environmental Engineering, University of Patras, Agrinio, Greece. ${ }^{6}$ Insect Pest Control Laboratory, Joint FAO/IAEA Division of Nuclear Techniques in Food and Agriculture, Vienna, Austria. ${ }^{7}$ Inscripta, Inc., 5500 Central Avenue \#220, Boulder, CO 80301, USA. ${ }^{8}$ Hawkesbury Institute for the Environment, Western Sydney University, Richmond, NSW 2753, Australia.

Received: 12 October 2019 Accepted: 13 March 2020

Published online: 30 March 2020

\section{References}

1. Adams MD, Celniker SE, Holt RA, Evans CA, Gocayne JD, Amanatides PG, Scherer SE, Li PW, Hoskins RA, Galle RF, et al. The genome sequence of Drosophila melanogaster. Science. 2000;287:2185-95.

2. Holt RA, Subramanian GM, Halpern A, Sutton GG, Charlab R, Nusskern DR, Wincker P, Clark AG, Ribeiro JM, Wides $R$, et al. The genome sequence of the malaria mosquito Anopheles gambiae. Science. 2002;298:129-49.

3. i5K Consortium. The i5K Initiative: advancing arthropod genomics for knowledge, human health, agriculture, and the environment. J Hered. 2013; 104:595-600.

4. Poelchau M, Childers C, Moore G, Tsavatapalli V, Evans J, Lee CY, Lin H, Lin JW, Hackett K. The i5k workspace@NAL--enabling genomic data access, visualization and curation of arthropod genomes. Nucleic Acids Res. 2015; 43:D714-9.

5. Li F, Zhao X, Li M, He K, Huang C, Zhou Y, Li Z, Walters JR. Insect genomes: progress and challenges. Insect Mol Biol. 2019;28(6):739-58. https://www. ncbi.nlm.nih.gov/pubmed/31120160.

6. Kyrou K, Hammond AM, Galizi R, Kranjc N, Burt A, Beaghton AK, Nolan T, Crisanti A. A CRISPR-Cas9 gene drive targeting doublesex causes complete population suppression in caged Anopheles gambiae mosquitoes. Nat Biotechnol. 2018;36:1062-6.

7. Vogel E, Santos D, Mingels L, Verdonckt TW, Broeck JV. RNA interference in insects: protecting Beneficials and controlling pests. Front Physiol. 2018;9: 1912

8. Weetman D, Wilding CS, Neafsey DE, Müller P, Ochomo E, Isaacs AT, Steen K, Rippon EJ, Morgan JC, Mawejje HD, et al. Candidate-gene based GWAS identifies reproducible DNA markers for metabolic pyrethroid resistance from standing genetic variation in East African Anopheles gambiae. Sci Rep. 2018;8(1):2920.

9. Clarkson CS, Temple HJ, Miles A. The genomics of insecticide resistance: insights from recent studies in African malaria vectors. Curr Opin Insect Sci. 2018;27:111-5.

10. Kotsakiozi P, Evans BR, Gloria-Soria A, Kamgang B, Mayanja M, Lutwama J, Le Goff G, Ayala D, Paupy C, Badolo A, et al. Population structure of a vector of human diseases: Aedes aegypti in its ancestral range, Africa. Ecol Evol. 2018; 8:7835-48.

11. Lee $Y$, Schmidt H, Collier TC, Conner WR, Hanemaaijer MJ, Slatkin M, Marshall JM, Chiu JC, Smartt CT, Lanzaro GC, et al. Genome-wide divergence among invasive populations of Aedes aegypti in California. BMC Genomics. 2019;20:204.

12. Gloss AD, Groen SC, Whiteman NK. A genomic perspective on the generation and maintenance of genetic diversity in herbivorous insects. Annu Rev Ecol Evol Syst. 2016;47:165-87.

13. Richards S, Murali SC. Best practices in insect genome sequencing: what works and what Doesn't. Curr Opin Insect Sci. 2015;7:1-7. 
14. Bentley DR, Balasubramanian S, Swerdlow HP, Smith GP, Milton J, Brown CG, Hall KP, Evers DJ, Barnes CL, Bignell HR, et al. Accurate whole human genome sequencing using reversible terminator chemistry. Nature. 2008; 456:53-9.

15. Loman NJ, Quick J, Simpson JT. A complete bacterial genome assembled de novo using only nanopore sequencing data. Nat Methods. 2015;12:733-5.

16. Phillippy AM. New advances in sequence assembly. Genome Res. 2017;27: xi-xiii.

17. Wick RR, Judd LM, Gorrie CL, Holt KE. Completing bacterial genome assemblies with multiplex MinION sequencing. Microb Genom. 2017;3: e000132.

18. Kitzman JO. Haplotypes drop by drop. Nat Biotechnol. 2016;34:296-8.

19. Zheng GX, Lau BT, Schnall-Levin M, Jarosz M, Bell JM, Hindson CM, Kyriazopoulou-Panagiotopoulou S, Masquelier DA, Merrill L, Terry JM, et al. Haplotyping germline and cancer genomes with high-throughput linkedread sequencing. Nat Biotechnol. 2016;34:303-11.

20. Weisenfeld NI, Kumar V, Shah P, Church DM, Jaffe DB. Direct determination of diploid genome sequences. Genome Res. 2017;27:757-67.

21. Matthews BJ, Dudchenko O, Kingan SB, Koren S, Antoshechkin I, Crawford JE, Glassford WJ, Herre M, Redmond SN, Rose NH, et al. Improved reference genome of Aedes aegypti informs arbovirus vector control. Nature. 2018; 563:501-7.

22. Talsania K, Mehta M, Raley C, Kriga Y, Gowda S, Grose C, Drew M, Roberts V, Cheng KT, Burkett S, et al. Genome assembly and annotation of the Trichoplusia ni Tni-FNL insect cell line enabled by long-read technologies. Genes (Basel). 2019; 10(2):E79. https://www.ncbi.nlm.nih.gov/pubmed/30678108.

23. Tsoumani KT, Mathiopoulos KD. Genome size estimation with quantitative real-time PCR in two Tephritidae species: Ceratitis capitata and Bactrocera oleae. J Appl Entomol. 2012;136:626-31.

24. Daane KM, Johnson MW. Olive fruit fly: managing an ancient pest in modern times. Annu Rev Entomol. 2010;55:151-69.

25. Montiel Bueno A, Jones O. Alternative methods for controlling the olive fly, Bactrocera oleae, involving semiochemicals. Bulletin OILB/SROP. 2002;25: 147-55.

26. Tsoumani KT, Augustinos AA, Kakani EG, Drosopoulou E, Mavragani-Tsipidou P, Mathiopoulos KD. Isolation, annotation and applications of expressed sequence tags from the olive fly, Bactrocera oleae. Mol Gen Genomics. 2011;285:33-45.

27. Pavlidi N, Dermauw W, Rombauts S, Chrysargyris A, Chrisargiris A, Van Leeuwen T, Vontas J. Analysis of the olive fruit Fly Bactrocera oleae Transcriptome and phylogenetic classification of the major detoxification gene families. PLoS One. 2013;8:e66533.

28. Sagri E, Reczko M, Gregoriou ME, Tsoumani KT, Zygouridis NE, Salpea KD, Zalom FG, Ragoussis J, Mathiopoulos KD. Olive fly transcriptomics analysis implicates energy metabolism genes in spinosad resistance. BMC Genomics. 2014;15:714

29. Sagri E, Reczko M, Tsoumani KT, Gregoriou ME, Harokopos V, Mavridou AM, Tastsoglou S, Athanasiadis K, Ragoussis J, Mathiopoulos KD. The molecular biology of the olive fly comes of age. BMC Genet. 2014;15(Suppl 2):S8

30. Tsoumani KT, Drosopoulou E, Mavragani-Tsipidou P, Mathiopoulos KD Molecular characterization and chromosomal distribution of a speciesspecific transcribed centromeric satellite repeat from the olive fruit fly, Bactrocera oleae. PLoS One. 2013;8:e79393.

31. Gabrieli P, Gomulski LM, Bonomi A, Siciliano P, Scolari F, Franz G, Jessup A, Malacrida AR, Gasperi G. Interchromosomal duplications on the Bactrocera oleae $Y$ chromosome imply a distinct evolutionary origin of the sex chromosomes compared to Drosophila. PLoS One. 2011:6:e17747.

32. Locke J, McDermid HE. Analysis of Drosophila chromosome 4 using pulsed field gel electrophoresis. Chromosoma. 1993;102:718-23.

33. Lohe AR, Hilliker AJ, Roberts PA. Mapping simple repeated DNA sequences in heterochromatin of Drosophila melanogaster. Genetics. 1993;134:1149-74.

34. Tomaszkiewicz M, Medvedev P, Makova KD. Y and W Chromosome Assemblies: Approaches and Discoveries. Trends Genet. 2017;33:266-82.

35. Koerich LB, Wang X, Clark AG, Carvalho AB. Low conservation of gene content in the Drosophila Y chromosome. Nature. 2008;456:949-51.

36. Willhoeft $U$, Franz $\mathrm{G}$. Identification of the sex-determining region of the Ceratitis capitata Y chromosome by deletion mapping. Genetics. 1996;144:737-45.

37. Saccone G, Pane A, Polito LC. Sex determination in flies, fruitflies and butterflies. Genetica. 2002;116:15-23.

38. Meccariello A, Salvemini M, Primo P, Hall B, Koskinioti P, Dalikova M, Gravina A, Gucciardino MA, Forlenza F, Gregoriou ME, et al. Maleness-on-the-Y
(MoY) orchestrates male sex determination in major agricultural fruit fly pests. Science. 2019;365(6460):1457-60. https://www.ncbi.nlm.nih.gov/ pubmed/31467189.

39. Knipling EF. Possibilities of insect control or eradication through the use of sexually sterile Males1. J Econ Entomol. 1955;48:459-62.

40. Franz G. Genetic Sexing Strains in Mediterranean Fruit Fly, an Example for Other Species Amenable to Large-Scale Rearing for the Sterile Insect Technique. In: HJ DVA, Robinson A, editors. Sterile Insect Technique. Dordrecht: Springer; 2005. p. 427-51.

41. Boisvert S, Raymond F, Godzaridis E, Laviolette F, Corbeil J. Ray Meta: scalable de novo metagenome assembly and profiling. Genome Biol. 2012;13:R122.

42. Boetzer M, Henkel CV, Jansen HJ, Butler D, Pirovano W. Scaffolding preassembled contigs using SSPACE. Bioinformatics. 2011;27:578-9.

43. Gurevich A, Saveliev V, Vyahhi N, Tesler G. QUAST: quality assessment tool for genome assemblies. Bioinformatics. 2013;29:1072-5.

44. Papanicolaou A, Schetelig MF, Arensburger P, Atkinson PW, Benoit JB, Bourtzis K, Castanera P, Cavanaugh JP, Chao H, Childers C, et al. The whole genome sequence of the Mediterranean fruit fly, Ceratitis capitata (Wiedemann), reveals insights into the biology and adaptive evolution of a highly invasive pest species. Genome Biol. 2016;17:192

45. Zygouridis NE, Argov Y, Nemny-Lavy E, Augustinos AA, Nestel D, Mathiopoulos KD. Genetic changes during laboratory domestication of an olive fly SIT strain. J Appl Entomol. 2014;138:423-32.

46. Loman NJ, Watson M. Successful test launch for nanopore sequencing. Nat Methods. 2015;12:303-4

47. Marcais G, Kingsford C. A fast, lock-free approach for efficient parallel counting of occurrences of k-mers. Bioinformatics. 2011;27:764-70.

48. Gregory TR: Animal Genome Size Database. Available online at: http://www. genomesize.com. 2005.

49. Hall AB, Qi Y, Timoshevskiy V, Sharakhova MV, Sharakhov IV, Tu Z. Six novel $Y$ chromosome genes in Anopheles mosquitoes discovered by independently sequencing males and females. BMC Genomics. 2013;14:273.

50. Drosopoulou E, Nakou I, Mavragani-Tsipidou P. The Bactrocera oleae genome: localization of nine genes on the polytene chromosomes of the olive fruit fly (Diptera: Tephritidae). Genome. 2014;57:573-6.

51. Carvalho AB, Clark AG. Efficient identification of $Y$ chromosome sequences in the human and Drosophila genomes. Genome Res. 2013;23:1894-907.

52. Mavragani-Tsipidou P, Karamanlidou G, Zacharopoulou A, Koliais S, Kastritisis C. Mitotic and polytene chromosome analysis in Dacus oleae (Diptera: Tephritidae). Genome. 1992:35:373-8.

53. Augustinos AA, Stratikopoulos EE, Drosopoulou E, Kakani EG, MavraganiTsipidou P, Zacharopoulou A, Mathiopoulos KD. Isolation and characterization of microsatellite markers from the olive fly, Bactrocera oleae, and their cross-species amplification in the Tephritidae family. BMC Genomics. 2008:9:618.

54. Zambetaki A, Kleanthous K, Mavragani-Tsipidou P. Cytogenetic analysis of Malpighian tubule and salivary gland polytene chromosomes of Bactrocera oleae (Dacus oleae) (Diptera: Tephritidae). Genome. 1995;38:1070-81.

55. Li H. Minimap2: pairwise alignment for nucleotide sequences. Bioinformatics. 2018;34:3094-100

56. Simao FA, Waterhouse RM, loannidis P, Kriventseva EV, Zdobnov EM. BUSCO: assessing genome assembly and annotation completeness with single-copy orthologs. Bioinformatics. 2015;31:3210-2.

57. BBMap [sourceforge.net/projects/bbmap/ Accessed 02 Feb 2020].

58. Vurture GW, Sedlazeck FJ, Nattestad M, Underwood CJ, Fang H, Gurtowski J, Schatz MC. GenomeScope: fast reference-free genome profiling from short reads. Bioinformatics. 2017:33:2202-4

59. Conesa A, Madrigal P, Tarazona S, Gomez-Cabrero D, Cervera A, McPherson A, Szczesniak MW, Gaffney DJ, Elo LL, Zhang X, Mortazavi A. A survey of best practices for RNA-seq data analysis. Genome Biol. 2016;17:13.

60. Kounatidis I, Crotti E, Sapountzis P, Sacchi L, Rizzi A, Chouaia B, Bandi C, Alma A, Daffonchio D, Mavragani-Tsipidou P, Bourtzis K. Acetobacter tropicalis is a major symbiont of the olive fruit fly (Bactrocera oleae). Appl Environ Microbiol. 2009;75:3281-8.

61. Mc CB. The origin and behavior of mutable loci in maize. Proc Natl Acad Sc U S A. 1950;36:344-55.

62. Wicker T, Sabot F, Hua-Van A, Bennetzen JL, Capy P, Chalhoub B, Flavell A, Leroy $\mathrm{P}$, Morgante $\mathrm{M}$, Panaud $\mathrm{O}$, et al. A unified classification system for eukaryotic transposable elements. Nat Rev Genet. 2007:8:973-82.

63. Maumus F, Fiston-Lavier A-S, Quesneville H. Impact of transposable elements on insect genomes and biology. Curr Opin Insect Sci. 2015;7:30-6. 
64. Kaminker JS, Bergman CM, Kronmiller B, Carlson J, Svirskas R, Patel S, Frise E, Wheeler DA, Lewis SE, Rubin GM, et al. The transposable elements of the Drosophila melanogaster euchromatin: a genomics perspective. Genome Biol. 2002;3:Research0084.

65. Bergman CM, Quesneville H, Anxolabehere D, Ashburner M. Recurrent insertion and duplication generate networks of transposable element sequences in the Drosophila melanogaster genome. Genome Biol. 2006;7:R112.

66. Goerner-Potvin P, Bourque G. Computational tools to unmask transposable elements. Nat Rev Genet. 2018;19:688-704.

67. Berthelier J, Casse N, Daccord N, Jamilloux V, Saint-Jean B, Carrier G. A transposable element annotation pipeline and expression analysis reveal potentially active elements in the microalga Tisochrysis lutea. BMC Genomics. 2018:19:378.

68. Arensburger $P$, Piegu B, Bigot $Y$. The future of transposable element annotation and their classification in the light of functional genomics what we can learn from the fables of Jean de la Fontaine? Mob Genet Elements. 2016;6:e1256852.

69. Hoede C, Arnoux S, Moisset M, Chaumier T, Inizan O, Jamilloux V, Quesneville H. PASTEC: an automatic transposable element classification tool. PLoS One. 2014;9:e91929.

70. Flutre T, Duprat E, Feuillet C, Quesneville H. Considering transposable element diversification in de novo annotation approaches. PLoS One. 2011; 6:e16526.

71. Clark AG, Eisen MB, Smith DR, Bergman CM, Oliver B, Markow TA, Kaufman TC, Kellis M, Gelbart W, lyer VN, et al. Evolution of genes and genomes on the Drosophila phylogeny. Nature. 2007;450:203-18.

72. Sessegolo C, Burlet N, Haudry A. Strong phylogenetic inertia on genome size and transposable element content among 26 species of flies. Biol Lett. 2016; 12(8):20160407. https://www.ncbi.nlm.nih.gov/pmc/articles/PMC5014035/.

73. Brillet B, Bigot $Y$, Auge-Gouillou C. Assembly of the TCl and mariner transposition initiation complexes depends on the origins of their transposase DNA binding domains. Genetica. 2007;130:105-20.

74. Peccoud J, Loiseau V, Cordaux R, Gilbert C. Massive horizontal transfer of transposable elements in insects. Proc Natl Acad Sci U S A. 2017;114:4721-6.

75. Loukeris TG, Livadaras I, Arca B, Zabalou S, Savakis C. Gene transfer into the medfly, Ceratitis capitata, with a Drosophila hydei transposable element. Science. 1995;270:2002-5.

76. Handler AM, McCombs SD, Fraser MJ, Saul SH. The lepidopteran transposon vector, piggyBac, mediates germ-line transformation in the Mediterranean fruit fly. Proc Natl Acad Sci U S A. 1998;95:7520-5.

77. Grabherr MG, Haas BJ, Yassour M, Levin JZ, Thompson DA, Amit I, Adiconis $X$, Fan L, Raychowdhury R, Zeng Q, et al. Full-length transcriptome assembly from RNA-seq data without a reference genome. Nat Biotechnol. 2011;29: 644-52.

78. [https://github.com/genomecuration/JAMg Accessed 19 Aug 2019].

79. Ryner LC, Goodwin SF, Castrillon DH, Anand A, Villella A, Baker BS, Hall JC, Taylor BJ, Wasserman SA. Control of male sexual behavior and sexual orientation in Drosophila by the fruitless gene. Cell. 1996;87:1079-89.

80. Conesa A, Gotz S, Garcia-Gomez JM, Terol J, Talon M, Robles M. Blast2GO: a universal tool for annotation, visualization and analysis in functional genomics research. Bioinformatics. 2005;21:3674-6.

81. Jones $P$, Binns D, Chang HY, Fraser M, Li W, McAnulla C, McWilliam H, Maslen J, Mitchell A, Nuka G, et al. InterProScan 5: genome-scale protein function classification. Bioinformatics. 2014;30:1236-40.

82. Nardi F, Carapelli A, Dallai R, Frati F. The mitochondrial genome of the olive fly Bactrocera oleae: two haplotypes from distant geographical locations. Insect Mol Biol. 2003;12:605-11.

83. Leimeister CA, Schellhorn J, Dorrer S, Gerth M, Bleidorn C, Morgenstern B. Prot-SpaM: fast alignment-free phylogeny reconstruction based on wholeproteome sequences. Gigascience. 2019;8(3):giy148. https://www.ncbi.nlm. nih.gov/pubmed/30535314.

84. Saitou N, Nei M. The neighbor-joining method: a new method for reconstructing phylogenetic trees. Mol Biol Evol. 1987;4:406-25.

85. Boc A, Diallo AB, Makarenkov V. T-REX: a web server for inferring, validating and visualizing phylogenetic trees and networks. Nucleic Acids Res. 2012;40:W573-9.

86. Letunic I, Bork P. Interactive tree of life (iTOL) v3: an online tool for the display and annotation of phylogenetic and other trees. Nucleic Acids Res. 2016:44:W242-5.

87. Emms DM, Kelly S. OrthoFinder: phylogenetic orthology inference for comparative genomics. Genome Biol. 2019;20(1):238. https://www.ncbi.nlm. nih.gov/pubmed/31727128.
88. Bardou P, Mariette J, Escudie F, Djemiel C, Klopp C. jvenn: an interactive Venn diagram viewer. BMC Bioinformatics. 2014;15:293.

89. Hanife G. Embryonic development of the olive fruit fly, Bactrocera oleae Rossi (Diptera: Tephritidae), in vivo. Turkish J Zool. 2014;38:598-602.

90. Genç Hanife NJL. Survival and development of Bactrocera oleae Gmelin (Diptera:Tephritidae) immature stages at four temperatures in the laboratory. Afr J Biotechnol. 2008;7:6.

91. Wigglesworth VB. Factors controlling Moulting and 'metamorphosis' in an insect. Nature. 1934;133:725-6.

92. Huber R, Hoppe W. Zur Chemie des Ecdysons, VII: Die Kristall- und Molekülstrukturanalyse des Insektenverpuppungshormons Ecdyson mit der automatisierten Faltmolekülmethode. Chemische Berichte. 1965;98:2403-24.

93. Buszczak M, Segraves WA. Insect metamorphosis: out with the old, in with the new. Curr Biol. 2000;10:R830-3.

94. Li B, Dewey CN. RSEM: accurate transcript quantification from RNA-seq data with or without a reference genome. BMC Bioinformatics. 2011;12:323.

95. Owens NDL, Blitz IL, Lane MA, Patrushev I, Overton JD, Gilchrist MJ, Cho KWY, Khokha MK. Measuring absolute RNA copy numbers at high temporal resolution reveals Transcriptome kinetics in development. Cell Rep. 2016;14:632-47.

96. McDowell IC, Manandhar D, Vockley CM, Schmid AK, Reddy TE, Engelhardt BE. Clustering gene expression time series data using an infinite Gaussian process mixture model. PLoS Comput Biol. 2018;14:e1005896.

97. Reimand J, Kull M, Peterson H, Hansen J, Vilo J. g:Profiler--a web-based toolset for functional profiling of gene lists from large-scale experiments. Nucleic Acids Res. 2007;35:W193-200.

98. Bayega A, Fahiminiya S, Oikonomopoulos S, Ragoussis J. Current and future methods for mRNA analysis: a drive toward single molecule sequencing. Methods Mol Biol. 2018;1783:209-41.

99. Lieberman-Aiden E, van Berkum NL, Williams L, Imakaev M, Ragoczy T, Telling A, Amit I, Lajoie BR, Sabo PJ, Dorschner MO, et al. Comprehensive mapping of long-range interactions reveals folding principles of the human genome. Science. 2009;326:289-93.

100. Dovetail Genomics: Overview of the Dovetail ${ }^{\mathrm{TM}}$ De Novo Assembly Process [https://dovetailgenomics.com/ga_tech_overview/. Accessed 9 Aug 2019].

101. Putnam NH, O'Connell BL, Stites JC, Rice BJ, Blanchette M, Calef R, Troll CJ, Fields A, Hartley PD, Sugnet CW, et al. Chromosome-scale shotgun assembly using an in vitro method for long-range linkage. Genome Res. 2016;26:342-50.

102. Mahajan S, Wei KH, Nalley MJ, Gibilisco L, Bachtrog D. De novo assembly of a young Drosophila Y chromosome using single-molecule sequencing and chromatin conformation capture. PLoS Biol. 2018;16:e2006348.

103. Ben-Yosef M, Aharon Y, Jurkevitch E, Yuval B. Give us the tools and we will do the job: symbiotic bacteria affect olive fly fitness in a diet-dependent fashion. Proc Biol Sci. 2010;277:1545-52.

104. Drew Dick YB. The evolution of fruit fly feeding behavior. In: NAL AM, editor. Fruit flies (Tephritidae): phylogeny and evolution of behavior. Boca Raton: CRC Press; 2000. p. 731-49.

105. Forister ML, Dyer LA, Singer MS, Stireman JO 3rd, Lill JT. Revisiting the evolution of ecological specialization, with emphasis on insect-plant interactions. Ecology. 2012;93:981-91.

106. Simon J-C, d'Alençon E, Guy E, Jacquin-Joly E, Jaquiéry J, Nouhaud $P$, Peccoud J, Sugio A, Streiff R. Genomics of adaptation to host-plants in herbivorous insects. Brief Funct Genomics. 2015;14:413-23.

107. White MJ. Cytological evidence on the phylogeny and classification of the Diptera. Evolution. 1949;3:252-61.

108. Bachtrog D. Y-chromosome evolution: emerging insights into processes of Y-chromosome degeneration. Nat Rev Genet. 2013;14:113-24.

109. Krsticevic FJ, Schrago CG, Carvalho AB. Long-Read Single Molecule Sequencing to Resolve Tandem Gene Copies: The Mst77Y Region on the Drosophila melanogaster Y Chromosome. G3 (Bethesda). 2015;5:1145-50.

110. Carvalho AB, Vicoso B, Russo CA, Swenor B, Clark AG. Birth of a new gene on the $Y$ chromosome of Drosophila melanogaster. Proc Natl Acad Sci U S A. 2015;112:12450-5.

111. Krzywinski J, Nusskern DR, Kern MK, Besansky NJ. Isolation and characterization of $Y$ chromosome sequences from the African malaria mosquito Anopheles gambiae. Genetics. 2004;166:1291-302.

112. Hughes JF, Skaletsky H, Brown LG, Pyntikova T, Graves T, Fulton RS, Dugan S, Ding $Y$, Buhay $C J$, Kremitzki C, et al. Strict evolutionary conservation followed rapid gene loss on human and rhesus $Y$ chromosomes. Nature. 2012:483:82-6.

113. Carvalho AB, Dobo BA, Vibranovski MD, Clark AG. Identification of five new genes on the $Y$ chromosome of Drosophila melanogaster. Proc Natl Acad Sci U S A. 2001;98:13225-30. 
114. Reisner W, Larsen NB, Silahtaroglu A, Kristensen A, Tommerup N, Tegenfeldt $\mathrm{JO}$, Flyvbjerg $\mathrm{H}$. Single-molecule denaturation mapping of DNA in nanofluidic channels. Proc Natl Acad Sci U S A. 2010;107:13294-9.

115. van Berkum NL, Lieberman-Aiden E, Williams L, Imakaev M, Gnirke A, Mirny LA, Dekker J, Lander ES. Hi-C: a method to study the three-dimensional architecture of genomes. J Vis Exp. 2010;39(39):1869. https://www.ncbi.nIm. nih.gov/pubmed/20461051.

116. Tomaszkiewicz M, Rangavittal S, Cechova M, Campos Sanchez R, Fescemyer HW, Harris R, Ye D, O'Brien PC, Chikhi R, Ryder OA, et al: A time- and costeffective strategy to sequence mammalian $Y$ Chromosomes: an application to the de novo assembly of gorilla Y. Genome Res. 2016;26:530-40.

117. Choo A, Nguyen TNM, Ward CM, Chen IY, Sved J, Shearman D, Gilchrist AS, Crisp P, Baxter SW. Identification of Y-chromosome scaffolds of the Queensland fruit fly reveals a duplicated gyf gene paralogue common to many Bactrocera pest species. Insect Mol Biol. 2019;28(6):873-86. https:// www.ncbi.nlm.nih.gov/pubmed/31150140.

118. Chang C-H, Larracuente AM. Heterochromatin-Enriched Assemblies Revea the Sequence and Organization of the \&lt;em\&gt;Drosophila melanogaster\&lt;/em\&gt; Y Chromosome. Genetics. 2019;211:333.

119. Tzanakakis ME, Economopoulos AP, Tsitsipis JA. The importance of conditions during the adult stage in evaluating an artificial food larvae of Dacus oleae (Gmelin) (Diptera: Tephritidae). Z Angew Entomol. 1967;59:127-30.

120. Zhang M, Zhang Y, Scheuring CF, Wu CC, Dong JJ, Zhang HB. Preparation of megabase-sized DNA from a variety of organisms using the nuclei method for advanced genomics research. Nat Protoc. 2012;7:467-78.

121. Koren S, Walenz BP, Berlin K, Miller JR, Bergman NH, Phillippy AM. Canu: scalable and accurate long-read assembly via adaptive k-mer weighting and repeat separation. Genome Res. 2017;27:722-36.

122. Walker BJ, Abeel T, Shea T, Priest M, Abouelliel A, Sakthikumar S, Cuomo CA, Zeng Q, Wortman J, Young SK, Earl AM. Pilon: an integrated tool for comprehensive microbial variant detection and genome assembly improvement. PLoS One. 2014;9:e112963.

123. Kurtz S, Phillippy A, Delcher AL, Smoot M, Shumway M, Antonescu C, Salzberg SL. Versatile and open software for comparing large genomes. Genome Biol. 2004;5:R12.

124. Untergasser A, Cutcutache I, Koressaar T, Ye J, Faircloth BC, Remm M, Rozen SG. Primer3--new capabilities and interfaces. Nucleic Acids Res. 2012;40:e115.

125. Drosopoulou E, Nakou I, Sichova J, Kubickova S, Marec F, MavraganiTsipidou P. Sex chromosomes and associated rDNA form a heterochromatic network in the polytene nuclei of Bactrocera oleae (Diptera: Tephritidae). Genetica. 2012;140:169-80.

126. Mavragani-Tsipidou P. ZA, Drosopoulou E. A, A. A., Bourtzis K. MF: Tephritid Fruit Flies (Diptera). In Protocols for Cytogenetic Mapping of Arthropod Genomes. Edited by V. SI. Boca Raton: CRC Press; 2014: 1-60.

127. RepeatMasker [http://www.repeatmasker.org/. Accessed 20 Jan 2018].

128. Han Y, Wessler SR. MITE-hunter: a program for discovering miniature inverted-repeat transposable elements from genomic sequences. Nucleic Acids Res. 2010;38:e199.

129. Yang L, Bennetzen JL. Structure-based discovery and description of plant and animal Helitrons. Proc Natl Acad Sci U S A. 2009;106:12832-7.

130. Ellinghaus D, Kurtz S, Willhoeft U. LTRharvest, an efficient and flexible software for de novo detection of LTR retrotransposons. BMC Bioinformatics. 2008:9:18.

131. Wenke T, Dobel T, Sorensen TR, Junghans H, Weisshaar B, Schmidt T. Targeted identification of short interspersed nuclear element families shows their widespread existence and extreme heterogeneity in plant genomes. Plant Cell. 2011;23:3117-28.

132. Rho M, Choi JH, Kim S, Lynch M, Tang H. De novo identification of LTR retrotransposons in eukaryotic genomes. BMC Genomics. 2007:8:90.

133. Price AL, Jones NC, Pevzner PA. De novo identification of repeat families in large genomes. Bioinformatics. 2005;21(Suppl 1):i351-8.

134. CD-HIT-EST [http://weizhongli-lab.org/cd-hit/. Accessed 18 Jul 2019].

135. Haas BJ, Papanicolaou A, Yassour M, Grabherr M, Blood PD, Bowden J, Couger MB, Eccles D, Li B, Lieber M, et al. De novo transcript sequence reconstruction from RNA-seq using the trinity platform for reference generation and analysis. Nat Protoc. 2013;8:1494-512

136. AH CTB, Zhang Q, Pyrkosz AB, Brom TH. A Reference-Free Algorithm for Computational Normalization of Shotgun Sequencing Data. ARXIV. 2012 1203:4802v2 [q-bio.GN].

\section{Publisher's Note}

Springer Nature remains neutral with regard to jurisdictional claims in published maps and institutional affiliations.
Ready to submit your research? Choose BMC and benefit from:

- fast, convenient online submission

- thorough peer review by experienced researchers in your field

- rapid publication on acceptance

- support for research data, including large and complex data types

- gold Open Access which fosters wider collaboration and increased citations

- maximum visibility for your research: over $100 \mathrm{M}$ website views per year

At $\mathrm{BMC}$, research is always in progress.

Learn more biomedcentral.com/submissions 